\title{
FINITE ELEMENT APPROXIMATIONS OF NONLINEAR ELASTIC WAVES
}

\author{
CHARALAMBOS G. MAKRIDAKIS
}

\begin{abstract}
In this paper we study finite element methods for a class of problems of nonlinear elastodynamics. We discretize the equations in space using Galerkin methods. For the temporal discretization, the construction of our schemes is based on rational approximations of $\cos x$ and $e^{x}$. We analyze semidiscrete as well as second- and fourth-order accurate in time fully discrete methods for the approximation of the solution of the problem and prove optimal-order $L^{2}$ error estimates. For some schemes a Taylor-type technique is used so that only linear systems of equations need be solved at each time step. In the proofs we need various estimates for a nonlinear elliptic projection, the proofs of which are also established in the paper.
\end{abstract}

\section{INTRODUCTION}

In this paper we shall study finite element methods for a class of problems of nonlinear elastodynamics. In particular, we consider the following initialboundary value problem: Let $\Omega$ be a bounded domain in $\mathbf{R}^{N}, N=1,2,3$, with smooth boundary $\partial \Omega(\Omega$ is viewed as the reference configuration of a homogeneous elastic body), and let $0<T<\infty$. We seek a displacement function $u: \bar{\Omega} \times[0, T] \rightarrow \mathbf{R}^{N}$ such that-index notation and the summation convention will be generally employed-

$$
\begin{aligned}
\ddot{u}_{i}(x, t) & =\partial_{\alpha} S_{i \alpha}(\nabla u(x, t))+f_{i}(x, t) \quad \text { in } \Omega \times[0, T], \\
u(x, t) & =0 \text { on } \partial \Omega \times[0, T], \\
u(x, 0) & =u^{0}(x), \quad \dot{u}(u, 0)=u^{1}(x) \text { in } \bar{\Omega},
\end{aligned}
$$

where dots denote differentiation with respect to $t$ and $\partial_{\alpha}=\partial / \partial x_{\alpha}$. Furthermore, $S$ is a given smooth $N \times N$ matrix-valued function defined on $\mathbf{R}^{N \times N}$ which characterizes the material-the Piola-Kirchhoff stress tensor-and $f$ is the body force. Also, $u^{0}$ and $u^{1}$ are given smooth functions which represent the initial displacement and the initial velocity, respectively. For a complete discussion of the physical background of the elasticity equations, cf., e.g., [15].

We shall discretize (1.1) in space using Galerkin methods. For the temporal discretization, since (1.1) is a wave-type equation, the construction of our schemes is based on rational approximations of $\cos x$ and $e^{x}$, cf. [1]-[3], [5]. We shall analyze semidiscrete as well as second- and fourth-order in time fully

Received by the editor June 3, 1991 and, in revised form, June 25, 1992.

1991 Mathematics Subject Classification. Primary 65M60, 73V05; Secondary 73D99.

Work supported in part by a research grant from the University of Crete. 
discrete methods for approximating the solution of (1.1). Before describing the results in detail, we proceed to introduce notation and list our assumptions for the problem.

We shall assume that the stress tensor $S$ has the following properties, cf. [10]:

$S$ is the gradient of a scalar-valued "stored energy function". Hence, if

$$
A_{i \alpha j \beta}(\eta):=\frac{\partial}{\partial \eta_{j \beta}} S_{i \alpha}(\eta), \quad \eta \in \mathbf{R}^{N \times N},
$$

we shall assume that the elasticities $A_{i \alpha j \beta}$ satisfy

$$
A_{i \alpha j \beta}=A_{j \beta i \alpha}, \quad 1 \leq i, \alpha, j, \beta \leq N .
$$

In addition, we assume that a strong ellipticity condition is satisfied in an open set $\mathscr{O}$ in the domain of $A_{i \alpha j \beta}$, i.e., that there is a positive constant $M_{1}$ such that

$$
A_{i \alpha j \beta}(\eta) \zeta_{\alpha} \zeta_{\beta} \xi_{i} \xi_{j} \geq M_{1}|\zeta|^{2}|\xi|^{2}
$$

for all $\eta \in \mathscr{O}$ and $\xi, \zeta \in \mathbf{R}^{N}$. Here, $|\cdot|$ denotes the Euclidean norm on $\mathbf{R}^{N}$.

Following standard notation, we let $W^{s, p}:=W^{s, p}(\Omega)^{N}$ be the usual Sobolev space of vector-valued functions whose generalized derivatives of order up to $s$ belong to $L^{p}(\Omega)^{N}$. We denote the corresponding norm by $\|\cdot\|_{s, p}$, by $|\cdot|_{s, p}$ the seminorm of order $s$, and put $H^{s}:=W^{s, 2}$ with norm $\|\cdot\|_{s}$. Also $(\cdot, \cdot)$, resp. $\|\cdot\|$, will denote the inner product, resp. norm, on $L^{2}:=L^{2}(\Omega)^{N}$ or $L^{2}(\Omega)^{N \times N}$, while $|\cdot|_{\infty}$ will be the norm on $L^{\infty}$. In addition, let $H_{0}^{1}$ be the subspace of $H^{1}$ consisting of the elements of $H^{1}$ that vanish on $\partial \Omega$ in the sense of trace.

Assuming (S1a, b), Dafermos and Hrusa [10], and Chen and v. Wahl [8], establish the existence of a unique local solution to the problem (1.1). In particular, the main result of [10] can be stated roughly as follows: Let $A_{i \alpha j \beta}$ and $f$ be sufficiently smooth and let $u^{0} \in H^{m}, u^{1} \in H^{m-1}$ for some integer $m \geq[N / 2]+3$. Assume further that $(\mathrm{S} 1 \mathrm{a}, \mathrm{b})$ hold, that the initial data satisfy the natural compatibility conditions of order $m$, i.e., that the initial values of time derivatives of $u$ up to order $m-1$ (as computed formally in terms of $u^{0}$ and $u^{1}$ using (1.1)) vanish on $\partial \Omega$, and that $\nabla u^{0}[\bar{\Omega}] \subset \mathscr{O}$. Then there is a $T>0$ for which (1.1) has a unique solution such that

$$
u \in \bigcap_{s=0}^{m} C^{m-s}\left([0, T] ; H^{s}\right) .
$$

By the Sobolev imbedding theorem the solution will be classical provided $m \geq$ $[N / 2]+3$. We shall therefore assume in the sequel that the above assumptions are fulfilled for $m$ sufficiently large to allow a unique solution $u$ of (1.1) to exist which is smooth enough for our purposes. Furthermore, we assume that there is an open convex set $\mathscr{M}$, with $\mathscr{\mathscr { M }} \subset \mathcal{O}$, such that $\nabla u[\bar{\Omega} \times[0, T]] \subset \mathscr{M}$ (see the proof of [10, Theorem 5.2]). If $\delta$ is the distance of $\mathscr{M}$ from $\partial \mathscr{O}$, and $\mathscr{M}_{\delta}:=\left\{\eta \in \mathbf{R}^{N \times N}: \inf _{\sigma \in \mathscr{M}}|\eta-\sigma| \leq \delta\right\}$, we let

$$
\mathrm{Z}:=\left\{\Phi \in L^{\infty}(\overline{\mathbf{\Omega}})^{N \times N}: \Phi(x) \in \mathscr{M}_{\delta}, x \in \bar{\Omega}\right\} .
$$


We will need $\mathbf{Z}$ in the error analysis; this is the set which is expected to contain the gradients of the approximations of $u$; thus, $(\mathbf{S} 2 \mathrm{~b})$ below will hold for these functions.

We now define the following forms:

$$
a(\phi, \psi)=\sum_{i, \alpha=1}^{N}\left(S_{i \alpha}(\nabla \phi), \partial_{\alpha} \psi_{i}\right), \quad \phi, \psi \in H^{1},
$$

and

$$
\tilde{a}(v ; \phi, \psi)=\sum_{i, \alpha, j, \beta=1}^{N}\left(A_{i \alpha j \beta}(\nabla v) \partial_{\beta} \phi_{j}, \partial_{\alpha} \psi_{i}\right), \quad v, \phi, \psi \in H^{1} .
$$

Using (S1) and the definition of $\mathbf{Z}$, we have

$$
\tilde{a}(v ; \phi, \psi)=\tilde{a}(v ; \psi, \phi), \quad v, \phi, \psi \in H^{1} .
$$

Further we assume that for $M_{0}>0$ we have

$$
\tilde{a}(v ; \phi, \phi) \geq M_{0}\|\nabla \phi\|^{2}, \quad v, \phi \in H^{1}, \nabla v \in \mathbf{Z} .
$$

(S2b) is a stronger assumption than (S1b). In particular (S1b) implies that $\tilde{a}(v ; \phi, \phi) \geq M_{0}\|\nabla \phi\|^{2}-\mu\|\phi\|^{2}, \mu \geq 0, v, \phi \in H^{1}, \nabla v \in \mathbf{Z}$. We note, however, that the techniques of this paper can be extended so that our results are valid under this weaker condition. A variational form of the problem (1.1) can now be stated as follows: Find $u(\cdot, t) \in H_{0}^{1}, 0 \leq t \leq T$, such that

$$
\begin{gathered}
(\ddot{u}(t), v)+a(u(t), v)=(f(t), v), \quad \forall v \in H_{0}^{1}, \\
u(x, 0)=u^{0}(x), \quad \dot{u}(x, 0)=u^{1}(x), \quad x \in \bar{\Omega} .
\end{gathered}
$$

Finite element discretization. We shall approximate the problem (1.2) by the finite element method, using, for the discretization in space, the usual piecewise polynomial shape functions, cf. e.g. $[9, \S 2.2]$. Specifically, for $0<h<1$ we assume that we are given a family $S_{h}$ of finite-dimensional subspaces of $W^{1, \infty} \cap H_{0}^{1}$ such that for some integer $r \geq 2$ and small $h$,

(i) $\inf _{\chi \in S_{h}}\left\{\|v-\chi\|+h\|v-\chi\|_{1}\right\} \leq C h^{s}\|v\|_{s}, \quad 1 \leq s \leq r, v \in H^{s} \cap H_{0}^{1}$.

Now let $A: H^{1} \rightarrow S_{h}$ be the nonlinear operator defined by

$$
(A v, \chi)=a(v, \chi), \quad \forall \chi \in S_{h} .
$$

Also, for given $v \in H^{1}$, we consider the linear operator $L(v): H^{1} \rightarrow S_{h}$ given by

$$
(L(v) \phi, \chi)=\tilde{a}(v ; \phi, \chi), \quad \forall \chi \in S_{h}, \phi \in H^{1} .
$$

Further, we assume that the following inverse inequalities hold:

(ii,a) There exists a positive constant $C_{0}$ such that for every $\chi \in S_{h}$

$$
\|\nabla \chi\| \leq C_{0} h^{-1}\|\chi\| \text { and }|\nabla \chi|_{\infty} \leq C_{0} h^{-1}|\chi|_{\infty} .
$$

Note that as a consequence of $(\mathbf{S} 2 \mathrm{~b})$ and the definition of $L(v)$ there exist positive constants $C_{1}, C_{2}$ such that

$$
C_{1}\|\nabla \chi\|^{2} \leq(L(v) \chi, \chi) \leq C_{2} h^{-2}\|\chi\|^{2}
$$

holds for all $v \in H^{1}$ with $\nabla v \in \mathbf{Z}$ and $\chi \in S_{h}$. 
(ii,b) There is a constant $C_{3}$ such that for every $\chi \in S_{h}$

$$
|\nabla \chi|_{\infty} \leq C_{3} h^{-N / 2}\|\nabla \chi\| \text { and }|\chi|_{\infty} \leq C_{3} h^{-N / 2}\|\chi\| .
$$

Let $P: L^{2} \rightarrow S_{h}$ denote the $L^{2}$-projection operator onto $S_{h}$. Then, a consequence of the assumption (i) is that there exists a constant $C$ such that

(iii)

$$
\|v-P v\| \leq C h^{s}\|v\|_{s}, \quad 1 \leq s \leq r, v \in H^{s} \cap H_{0}^{1} .
$$

We define now a nonlinear elliptic projection of the solution $u$ of (1.1), denoted by $W(t)$ (or $w(t)$ in $\S 4$ ) as follows. For $0 \leq t \leq T$, let $W(t) \in S_{h}$ be the solution of the nonlinear system

$$
a(W(t), \chi)=a(u(t), \chi), \quad \forall \chi \in S_{h} .
$$

It is known, cf. Dobrowolski and Rannacher [12], and Rannacher [19], that the equation (1.5) has a locally unique solution $W(t) \in S_{h}$ for $0 \leq t \leq T$. Furthermore, $W$ has the following approximation properties:

(iv,a) There exist constants $C_{s}(u)$ that depend on $u$ such that

$$
\|u(t)-W(t)\| \leq C_{s}(u) h^{s}, \quad 2 \leq s \leq r,
$$

and

$$
|u(t)-W(t)|_{\infty} \leq C_{s}(u) h^{s}|\log h|^{\theta(r)}, \quad 2 \leq s \leq r,
$$

where $\theta(r)=0$ if $r>2$ and $\theta(r)=\frac{N}{4}+1>0$ if $r=2$. (For a proof of these estimates, cf. [12] for $r \geq 2$ and [19] for $r=2$ with a different technique.)

In addition, we shall prove in $\S 5$ that for the time derivatives of $W$ there holds

(iv,b) $\left\|u^{(j)}(t)-W^{(j)}(t)\right\| \leq C_{s, j}(u) h^{s}, 2 \leq s \leq r, j=0,1, \ldots$.

Finally, we shall suppose that there exist constants $C_{j}$, independent of $h$, such that

(v) $\left\|W^{(j)}(t)\right\|_{1, \infty} \leq C_{j}, j=0,1$.

In $\S 5$ we shall establish that (i)-(iv) imply (v) under the restriction that $r-$ $N / 2-1 \geq 0$.

Summary of results. We consider first the semidiscrete analog of problem (1.2) on $S_{h}$, namely the problem of finding $u_{h}:[0, T] \rightarrow S_{h}$ such that

$$
\left(\ddot{u}_{h}, \chi\right)+a\left(u_{h}, \chi\right)=(f, \chi), \quad \forall \chi \in S_{h},
$$

given initial values $u_{h}(0)$ and $\dot{u}_{h}(0)$ approximating $u^{0}$ and $u^{1}$ in $S_{h}$.

In $\S 2$ we shall show that the problem (1.6) has a locally unique solution and that the optimal-order $L^{2}$ error estimate

$$
\max _{0 \leq t \leq T}\left\|u(t)-u_{h}(t)\right\| \leq C(u) h^{r}
$$

holds, under the assumption that $r-N / 2-1>0$ and for an appropriate choice of initial approximations.

For work on semidiscrete approximations to scalar nonlinear wave equations of the form $v_{t t}+\sum_{i=1}^{N} \partial_{i} F_{i}(\nabla v)=g$, cf. [11].

In $\S 3$ we construct two two-step fully discrete schemes to approximate (1.1) in time as well, that are based on second-order accurate approximations to the cosine. They are both second-order accurate with respect to the time step $k$. Computing the approximation $U^{n} \in S_{h}$ of $u\left(t_{n}\right), t_{n}=n k, n=0,1, \ldots, J$, 
$t_{J}=T$, with the first scheme requires the solution of a system of nonlinear equations for each $n$, while the second scheme only requires solving one linear system of equations for each $n$. Under certain smoothness assumptions on the solution of $(1.1)$, we show in $\S 3$ that these approximations satisfy

$$
\max _{0 \leq n \leq J}\left\|u\left(t_{n}\right)-U^{n}\right\| \leq C\left(h^{r}+k^{2}\right),
$$

under the restrictions:

$(\alpha) k=o\left(h^{1 / 2}\right)$ and $h^{r-1 / 2}=o(k)$, when $N=1$,

(B) $k=o(h), r>2$, and $h^{r-1}=o(k)$, when $N=2$ and

(r) $k=o\left(h^{3 / 2}\right), r>3$ and $h^{r-3 / 2}=o(k)$ when $N=3$.

In [6], Bales and Dougalis analyzed fully discrete fourth-order accurate in time cosine schemes for the nonlinear scalar wave equation

$$
v_{t t}-\sum_{i, j=1}^{N} \partial_{j}\left(a_{i j}(v) \partial_{i} v\right)+a_{0}(v)=g(v)
$$

and proved optimal-rate $L^{2}$ error estimates in space and time. Our general plan of error analysis in $\S 3$ follows that of [6]. We shall consider only secondorder accurate schemes since, owing to the special form of the equations of nonlinear elastodynamics, it is not clear how to construct useful fourth-order in time cosine-type schemes. Note that in [17] up to fourth-order accurate cosinetype schemes have been analyzed for the equation (1.7) in the more general case where the function $g$ depends on $u_{t}$ and $\nabla u$ as well.

In $\S 4$ we shall study single-step fully discrete methods for the approximation of $u$ that have temporal order of accuracy 2,3 , or 4 . In order to construct these schemes, we first write the semidiscrete problem (1.6) as a first-order in time system of ordinary differential equations, that we then discretize in time using methods based on rational approximations of the exponential, cf., e.g., [1, $3]$. With suitable choices we derive fully discrete schemes in which computing the approximations $\left(\begin{array}{l}U_{1}^{n} \\ U_{2}^{n}\end{array}\right) \approx\left(\begin{array}{l}u\left(t_{n}\right) \\ \dot{u}\left(t_{n}\right.\end{array}\right)$ needs only the solution of linear systems of equations at each time step. Moreover, in $\S 4$, we prove that if the quantity $k^{-1} h^{-N / 2}\left(h^{r}+k^{\nu}\right)$ remains small as $k, h \rightarrow 0$, then

$$
\max _{0 \leq n \leq J}\left(\left\|u\left(t_{n}\right)-U_{1}^{n}\right\|+\left\|\dot{u}\left(t_{n}\right)-U_{2}^{n}\right\|\right) \leq c\left(h^{r}+k^{\nu}\right), \quad \nu=2,3 \text { or } 4,
$$

i.e., that an optimal-order in space and time $L^{2}$ error estimate holds for the approximations of the displacement $u$ as well as of the velocity $\dot{u}$. (Note that the restriction that $k^{-1} h^{-N / 2}\left(h^{r}+k^{\nu}\right)$ remains small as $k, h \rightarrow 0$ follows, if $\nu=2$, from the mesh conditions $(\alpha),(\beta),(\gamma)$ stated previously. If $\nu=3$ or 4 , it follows e.g. from the weak mesh condition that $k^{\nu-1}=o\left(h^{N / 2}\right), h^{r-N / 2}=$ $o(k)$ provided that $r>\frac{N}{2}$.)

In [4], Bales considers the nonlinear scalar hyperbolic problem (1.7) and analyzes fully discrete schemes of up to fourth-order temporal accuracy that are generated by rational approximations of $e^{x}$. Under the restriction that $r \geq 3$ and $\nu \geq 3$ for $N=2,3$ he proves optimal-order $L^{2}$ error estimates for the approximations of $v$. The general plan of our error analysis in $\S 4$ follows that of [4], and also that of Bramble and Sammon [7], in which fully discrete approximations for parabolic problems are analyzed. However, in our analysis we use a stronger norm for the estimates. This preserves the optimal order of 
convergence, cf. Lemma 4.1 below, and also allows us to handle the effect of the presence of $\nabla u$ in the operator $S$ in a satisfactory manner. (Of course, we must require that $k^{-1} h^{-N / 2}\left(h^{r}+k^{\nu}\right)$ be small, a restriction inherent in the problem itself rather than in the techniques used for the error analysis, cf. the analogous restrictions in $\S \S 2$ and 3.) Note also that, if one applies our technique in the case of Bales' schemes for (1.7) one can prove the analogous estimates, but without the restrictions $r \geq 3, \nu \geq 3$.

\section{SEMIDISCRETE APPROXIMATIONS}

As stated in the Introduction, the continuous-in-time finite element approximation (semidiscrete approximation) $u_{h}:[0, T] \rightarrow S_{h}$ of the solution of (1.1) satisfies the following initial value problem in $S_{h}$ :

$$
\begin{gathered}
\left(\ddot{u}_{h}, \chi\right)+a\left(u_{h}, \chi\right)=(f, \chi), \quad \forall \chi \in S_{h}, \quad 0 \leq t \leq T, \\
u_{h}(0)=u_{h}^{0}, \quad \dot{u}_{h}(0)=u_{h}^{1} \in S_{h} .
\end{gathered}
$$

We have now the following result:

Theorem 2.1. Let $u$ be the solution of (1.1). We assume that $r-N / 2-1>0$ and that the initial values $u_{h}^{0}, u_{h}^{1} \in S_{h}$ have been chosen such that

$$
\left\|u_{h}^{0}-W(0)\right\|_{1}+\left\|u_{h}^{1}-\dot{W}(0)\right\| \leq c h^{r},
$$

where $W(t)$ is the solution of (1.5); then the semidiscrete problem (2.1) has a locally unique solution that satisfies, for a constant $c$ independent of $h$,

$$
\max _{0 \leq t \leq T}\left\|u_{h}(t)-u(t)\right\| \leq c h^{r}
$$

Proof. Let $u_{h}-u=\left(u_{h}-W\right)+(W-u)=: \theta+\rho$. Then, using (1.2), (1.5), and (2.1), we have

$$
(\ddot{\theta}, \chi)+a\left(u_{h}, \chi\right)-a(W, \chi)=-(\ddot{\rho}, \chi), \quad \forall \chi \in S_{h} .
$$

In the sequel we shall use the following formula (Taylor's theorem): If $\nabla v, \nabla w \in \mathbf{Z}$, there holds

$$
S_{i \alpha}(\nabla v)=S_{i \alpha}(\nabla w)+\sum_{j, \beta=1}^{N} \partial_{\beta}\left(v_{j}-w_{j}\right) \int_{0}^{1} \frac{\partial}{\partial \eta_{j \beta}} S_{i \alpha}(\nabla w+\tau(\nabla(v-w))) d \tau
$$

Since $\mathbf{Z}$ is convex, the term in the integral remainder is well defined.

Let us assume for a moment that $\nabla u_{h} \in \mathbf{Z}$. Then, since for $h$ sufficiently small, $\nabla W \in \mathbf{Z}$ as a consequence of (ii,b) and (iv,a), we have, using (2.4),

$$
a\left(u_{h}, \chi\right)-a(W, \chi)=\int_{0}^{1} \tilde{a}\left(W+\tau\left(u_{h}-W\right) ; \theta, \chi\right) d \tau, \quad \chi \in S_{h} .
$$

Hence, (2.3) takes the form

$$
(\ddot{\theta}, \chi)+\int_{0}^{1} \tilde{a}\left(W+\tau\left(u_{h}-W\right) ; \theta, \chi\right) d \tau=-(\ddot{\rho}, \chi) .
$$

Given $W=W(t)$, we consider a form defined for $\phi, \psi, \chi \in S_{h}$ (with $\nabla \phi \in \mathbf{Z}$ ) as follows:

$$
A(\phi ; \psi, \chi)=\int_{0}^{1} \tilde{a}(W+\tau(\phi-W) ; \psi, \chi) d \tau
$$


Consider the following subset of $Y:=C\left([0, T] ; H^{1}\right) \cap C^{1}\left([0, T] ; L^{2}\right)$ :

$$
\begin{aligned}
& \mathscr{I}=\left\{\psi:[0, T] \rightarrow S_{h},\right. \\
& \quad \text { where } \max _{0 \leq t \leq T}\left(\|\psi(t)-W(t)\|_{1}+\|\dot{\psi}(t)-\dot{W}(t)\|\right) \leq C_{*}(u) h^{r}, \\
& \quad \text { and } \nabla \psi(t) \in \mathbf{Z}, 0 \leq t \leq T\},
\end{aligned}
$$

where $C_{*}(u)=C_{*}$ is a positive constant which will be specified later. It is obvious that $\mathscr{I}$ is not empty, since $W \in \mathscr{I}$. Also, if a sequence $\left\{\psi_{n}\right\}_{n=1}^{\infty} \subset \mathscr{I}$ converges in $Y$ to $\psi \in Y$, we see easily that $\psi \in \mathscr{I}$. Hence, $\mathscr{I}$ is a closed subset of $Y$.

With this notation, define a mapping $\mathscr{N}$ on $\mathscr{I}$ as follows: If $\phi \in \mathscr{I}$, the image $\mathscr{N}(\phi)=: u_{\phi}$ is given by the relations

$$
u_{\phi}(0)=u_{h}^{0}, \quad \dot{u}_{\phi}(0)=u_{h}^{1}, \quad \text { for } t=0,
$$

and $u_{\phi}(t) \in S_{h}$, for $0<t \leq T$, such that

$$
\left(\ddot{\theta}_{\phi}, \chi\right)+A\left(\phi ; \theta_{\phi}, \chi\right)=-(\ddot{\rho}, \chi), \quad \forall \chi \in S_{h}, 0<t \leq T,
$$

where $\theta_{\phi}=u_{\phi}-W$. In order to complete the proof of the theorem, it suffices to show that $\mathscr{N}$ has a unique fixed point in $\mathscr{J}$. Indeed, if $v_{h}$ is this fixed point, then $v_{h}$ satisfies (2.5) and $\nabla v_{h} \in \mathbf{Z}$; therefore $v_{h}$ satisfies (2.3) too, i.e., is a solution of (2.1). Furthermore, since $v_{h} \in \mathscr{I}$, the approximation property (iv,a) implies that $\max _{0 \leq t \leq T}\left\|v_{h}(t)-u(t)\right\| \leq c h^{r}$.

We will establish the existence of a unique fixed point in $\mathscr{I}$ by showing that the pair $\mathscr{I}, \mathcal{N}$ satisfies the assumptions of Banach's fixed point theorem, namely that

(a) $\mathscr{N}(\mathscr{I}) \subset \mathscr{I}$

(b) $\mathscr{N}$ is a contraction with respect to $d(\cdot, \cdot)$,

where for $\phi, \psi \in \mathcal{F}, d(\phi, \psi):=\max _{0 \leq t \leq T}\left(\|\phi(t)-\psi(t)\|_{1}+\|\dot{\phi}(t)-\dot{\psi}(t)\|\right)$. (Note that we can show, by a similar argument as in the proof of $(b)$ below, that if $u_{h}$ and $\tilde{u}_{h}$ are two solutions of (2.1) with $\nabla u_{h}, \nabla \tilde{u}_{h} \in \mathbf{Z}$ and $\left\|u-u_{h}\right\|$, $\left\|u-\tilde{u}_{h}\right\| \leq C h^{r}$, then $u_{h}=\tilde{u}_{h}$.)

For (a), we first observe that $\mathscr{N}$ is well defined. Indeed, if $\phi \in \mathscr{I}$, since $\nabla W$ belongs to $\mathbf{Z}$, the element $W+\tau(\phi-W), 0 \leq \tau \leq 1$, is such that $\nabla(W+\tau(\phi-W)) \in \mathbf{Z}$, and the bilinear form $A(\phi ; \cdot, \cdot)$ is symmetric and positive definite. Hence, the relations (2.6) and (2.7) describe $u_{\phi}:[0, T] \rightarrow S_{h}$ uniquely as the solution of an initial value problem of a second-order system of ODEs.

Now putting $\chi=\dot{\theta}_{\phi}$ in (2.7), we have for $0 \leq t \leq T$

$$
\left(\ddot{\theta}_{\phi}, \dot{\theta}_{\phi}\right)+A\left(\phi ; \theta_{\phi}, \dot{\theta}_{\phi}\right)=-\left(\ddot{\rho}, \dot{\theta}_{\phi}\right),
$$

or

$$
\begin{aligned}
& \frac{1}{2} \frac{d}{d t}\left\{\left\|\dot{\theta}_{\phi}\right\|^{2}+A\left(\phi ; \theta_{\phi}, \theta_{\phi}\right)\right\} \\
& \quad=-\left(\ddot{\rho}, \dot{\theta}_{\phi}\right)+\sum_{i, \alpha, j, \beta=1}^{N} \frac{1}{2} \int_{0}^{1}\left(\partial_{t}\left[A_{i \alpha j \beta}(\nabla W+\tau \nabla(\phi-W))\right] \partial_{j} \theta_{\phi, \beta}, \partial_{i} \theta_{\phi, \alpha}\right) d \tau .
\end{aligned}
$$


Since $A(\phi ; \cdot, \cdot)$ is positive definite, integration yields for $0 \leq t \leq T$ (2.8)

$$
\begin{aligned}
\left\|\dot{\theta}_{\phi}(t)\right\|^{2} & +M_{0}\left\|\nabla \theta_{\phi}(t)\right\|^{2} \\
\leq & c\left(\left\|\dot{\theta}_{\phi}(0)\right\|^{2}+\left\|\theta_{\phi}(0)\right\|_{1}^{2}\right)+c \int_{0}^{t}\|\ddot{\rho}\|^{2} d s+\int_{0}^{t}\left\|\dot{\theta}_{\phi}\right\|^{2} d s \\
& +\sum_{i, \alpha, j, \beta=1}^{N} \frac{1}{2} \int_{0}^{t} \int_{0}^{1}\left(\partial_{t}\left[A_{i \alpha j \beta}(\nabla W+\tau \nabla(\phi-W))\right] \partial_{j} \theta_{\phi, \beta}, \partial_{i} \theta_{\phi, \alpha}\right) d \tau d s .
\end{aligned}
$$

From (iv,b) we have $\int_{0}^{t}\|\ddot{\rho}\|^{2} d s \leq c h^{2 r}$. Also, (2.2) and (2.6) give $\left\|\dot{\theta}_{\phi}(0)\right\|^{2}+$ $\left\|\theta_{\phi}(0)\right\|_{1}^{2} \leq c h^{2 r}$. Finally, to estimate the last term of (2.8), observe that

$$
\begin{aligned}
& \partial_{t}\left(A_{i \alpha j \beta}(\nabla W+\tau \nabla(\phi-W))\right) \\
& \quad=\sum_{k, \gamma=1}^{N} \frac{\partial A_{i \alpha j \beta}}{\partial \eta_{k \gamma}}(\nabla W+\tau \nabla(\phi-W)) \partial_{\gamma}\left(\dot{W}_{k}+\tau\left(\dot{\phi}_{k}-\dot{W}_{k}\right)\right) .
\end{aligned}
$$

Using the fact that the values of $\nabla W+\tau \nabla(\phi-W)$ lie in a bounded set of $\mathbf{R}^{N \times N}$, the smoothness of $A_{i \alpha j \beta}$, and (v), (ii,a,b), we have that

$$
\begin{aligned}
& \left|\sum_{i, \alpha, j, \beta}^{N} \frac{1}{2} \int_{0}^{1}\left(\partial_{t}\left[A_{i \alpha j \beta}(\nabla W+\tau \nabla(\phi-W))\right] \partial_{j} \theta_{\phi, \beta}, \partial_{i} \theta_{\phi, \alpha}\right) d \tau\right| \\
& \quad \leq c|\dot{W}|_{1, \infty}\left\|\theta_{\phi}\right\|_{1}^{2}+c|\dot{\phi}-\dot{W}|_{1, \infty}\left\|\theta_{\phi}\right\|_{1}^{2} \\
& \quad \leq c\left\|\theta_{\phi}\right\|_{1}^{2}+c h^{-1-N / 2}\|\dot{\phi}-\dot{W}\|\left\|\theta_{\phi}\right\|_{1}^{2} \leq c\left\|\theta_{\phi}\right\|_{1}^{2},
\end{aligned}
$$

where in the last inequality we used the fact that, since $r>1+N / 2, \phi \in \mathscr{J}$, we can choose an $h_{0}$ such that for $h<h_{0}$ we have $c h^{-1-N / 2}\|\dot{\phi}-\dot{W}\| \leq 1$ for $0 \leq t \leq T$.

Combining the above estimates in (2.8), we have

$$
\left\|\dot{\theta}_{\phi}(t)\right\|^{2}+M_{0}\left\|\nabla \theta_{\phi}(t)\right\|^{2} \leq c h^{2 r}+c \int_{0}^{t}\left(\left\|\dot{\theta}_{\phi}\right\|^{2}+\left\|\theta_{\phi}\right\|_{1}^{2}\right) d s .
$$

Hence, using Gronwall's lemma, we obtain that for some constant $C_{1}(u)$ independent of $h$

$$
\left\|\theta_{\phi}(t)\right\|_{1}+\left\|\dot{\theta}_{\phi}(t)\right\| \leq C_{1}(u) h^{r}, \quad 0 \leq t \leq T .
$$

Now tracing back constants through the previous estimates, we observe that the constant $C_{1}(u)$ does not depend on $C_{*}(u)$ and on $\phi$. Consequently, setting $C_{*}(u):=C_{1}(u)$ in the definition of $\mathscr{F}$, we have

$$
\left\|\theta_{\phi}(t)\right\|_{1}+\left\|\dot{\theta}_{\phi}(t)\right\| \leq C_{*}(u) h^{r}, \quad 0 \leq t \leq T .
$$

From (iv,a), (ii,a), we conclude that there is an $h_{1}>0$ such that for $h<h_{1}$ we have $|\nabla u(t) \rightarrow \nabla W(t)|_{\infty} \leq \delta / 2$. Consequently, choosing $h_{2}>0$ so that for $h<h_{2},\left|\nabla u_{\phi}(t)-\nabla W(t)\right|_{\infty} \leq c h^{-N / 2}\left\|\theta_{\phi}(t)\right\|_{1} \leq c C_{*}(u) h^{r-N / 2}<\delta / 2$, we obtain that $\nabla u_{\phi} \in \mathbf{Z}$. Hence, we establish the validity of (a) by choosing $h<h_{*}=\min \left\{h_{0}, h_{1}, h_{2}\right\}$.

For the proof of (b) let $R=\phi-\phi^{\prime}$ and $\Theta=u_{\phi}-u_{\phi^{\prime}}$, where $\phi, \phi^{\prime} \in \mathcal{I}$. Then (2.7) gives

$$
(\ddot{\Theta}, \chi)+A(\phi ; \Theta, \chi)=A\left(\phi^{\prime} ; \theta_{\phi^{\prime}}, \chi\right)-A\left(\phi ; \theta_{\phi^{\prime}}, \chi\right), \quad 0 \leq t \leq T .
$$


Letting $\chi=\dot{\Theta}$, in the same way as before we get

$$
\begin{aligned}
&\|\dot{\boldsymbol{\Theta}}(t)\|^{2}+M_{0}\|\nabla \boldsymbol{\Theta}(t)\|^{2} \\
& \leq \int_{0}^{t}\|\dot{\boldsymbol{\Theta}}\|^{2} d s+c \int_{0}^{t}\left\{A\left(\phi^{\prime} ; \theta_{\phi^{\prime}}, \dot{\boldsymbol{\Theta}}\right)-A\left(\phi ; \theta_{\phi^{\prime}}, \dot{\boldsymbol{\Theta}}\right)\right\} d s \\
&+c \sum_{i, \alpha, j, \beta=1}^{N} \frac{1}{2} \int_{0}^{t} \int_{0}^{1}\left(\partial_{t}\left[A_{i \alpha j \beta}(\nabla W+\tau \nabla(\phi-W))\right] \partial_{\beta} \boldsymbol{\Theta}_{j}, \partial_{\alpha} \boldsymbol{\Theta}_{i}\right) d \tau d s .
\end{aligned}
$$

For $h<h_{0}$ and $\phi \in \mathscr{I}$ we have

$$
\left|\sum_{i, \alpha, j, \beta}^{N} \frac{1}{2} \int_{0}^{1}\left(\partial_{t}\left[A_{i \alpha j \beta}(\nabla W+\tau \nabla(\phi-W))\right] \partial_{\beta} \boldsymbol{\Theta}_{j}, \partial_{\alpha} \boldsymbol{\Theta}_{i}\right) d \tau\right| \leq C\|\boldsymbol{\Theta}(t)\|_{1}^{2} .
$$

Now using (ii,a,b) and the fact that $A_{i \alpha j \beta}$ are smooth (and in particular Lipschitz), we have

$$
\begin{aligned}
\left|A\left(\phi^{\prime} ; \theta_{\phi^{\prime}}, \dot{\Theta}\right)-A\left(\phi ; \theta_{\phi^{\prime}}, \dot{\Theta}\right)\right| & \\
= & \mid \sum_{i, \alpha, j, \beta}^{N} \int_{0}^{1}\left(A_{i \alpha j \beta}\left(\nabla W+\tau \nabla\left(\phi^{\prime}-W\right)\right) \partial_{\beta} \theta_{\phi^{\prime}, j}, \partial_{\alpha} \dot{\Theta}_{i}\right) d \tau \\
& \quad-\sum_{i, \alpha, j, \beta}^{N} \int_{0}^{1}\left(A_{i \alpha j \beta}(\nabla W+\tau \nabla(\phi-W)) \partial_{\beta} \theta_{\phi^{\prime}, j}, \partial_{\alpha} \dot{\Theta}_{i}\right) d \tau \mid \\
& \leq c\left\|\nabla\left(\phi-\phi^{\prime}\right)\right\|\left|\theta_{\phi^{\prime}}\right|_{1, \infty}\|\nabla \dot{\Theta}\| \leq c \gamma(h)\|\nabla R\|\|\dot{\Theta}\|,
\end{aligned}
$$

where $\gamma(h)=h^{r-1-N / 2}$. In the last relation we used that $u_{\phi^{\prime}} \in \mathscr{I}$.

Combining the above estimates, and applying Gronwall's lemma in (2.9), we have, since $\boldsymbol{\Theta}(0)=\dot{\Theta}(0)=0$,

$$
\max _{0 \leq t \leq T}\left(\|\boldsymbol{\Theta}(t)\|_{1}+\|\dot{\boldsymbol{\Theta}}(t)\|\right) \leq \gamma(h) C_{2}(u) \max _{0 \leq t \leq T}\|R(t)\|_{1} .
$$

Since $r-1-N / 2>0$ we can choose an $h_{3}$ such that for $h<h_{3}$ we have $\gamma(h) C_{2}(u)=: L<1$; i.e., taking $h<\min \left\{h_{*}, h_{3}\right\}$, we see that (a), (b) hold, and so the proof of the theorem is complete.

\section{TWO-STEP FULLY DISCRETE SCHEMES OF SECOND-ORDER TEMPORAL ACCURACY}

In this section we shall construct two fully discrete schemes for the approximation of the solution of (1.4). These schemes are based on second-order accurate rational approximations to the cosine, cf. $[2,5,13]$. For real $x$ we consider a rational function of the form

$$
r(x)=\frac{1+p_{1} x^{2}}{1+q_{1} x^{2}}, \quad q_{1}>0,
$$

approximating $\cos x$. For accuracy purposes we assume that $p_{1}=q_{1}-1 / 2$, which implies that in general $r(x)=\cos x+O\left(x^{4}\right)$, as $x \rightarrow 0$. We shall also 
assume that $q_{1}>1 / 4$, which ensures that the stability condition $|r(x)| \leq 1$ is satisfied for all $x \in \mathbf{R}$. Let $k>0$ be the (constant) time step and $t_{n}=n k$, $n=0,1, \ldots, J, J k=T$. As a consequence of the second-order accuracy of $r(x)$, for every smooth function $y=y(t)$ on $[0, T]$ we have (with $y^{(j)}:=$ $\left.d^{j} y / d t^{j}\right)$

$$
\left(I-k^{2} q_{1} \partial_{t}^{2}\right)\left(y\left(t_{n+1}\right)+y\left(t_{n-1}\right)\right)=2\left(I-k^{2} p_{1} \partial_{t}^{2}\right) y\left(t_{n}\right)+O\left(k^{4} y^{(4)}\right)
$$

Motivated by the above relation and (1.4), we can now define the following fully discrete schemes. We first seek $V^{j} \in S_{h}, 0 \leq j \leq J$, approximating $u^{j}=$ $u\left(t_{j}\right)$ for $0 \leq j \leq J$ by defining recursively $V^{n+1} \in S_{h}$ for $n=1, \ldots, J-1$ as the solution of the nonlinear system of equations

$$
\begin{aligned}
\left(V^{n+1}-2 V^{n}\right. & \left.+V^{n-1}, \chi\right)+k^{2} q_{1} a\left(V^{n+1}, \chi\right) \\
& -2 k^{2} p_{1} a\left(V^{n}, \chi\right)+k^{2} q_{1} a\left(V^{n-1}, \chi\right) \\
= & k^{2}\left(q_{1} f^{n+1}-2 p_{1} f^{n}+q_{1} f^{n-1}, \chi\right), \quad \forall \chi \in S_{h},
\end{aligned}
$$

where $V^{0}, V^{1}$ will be given in $S_{h}$ and $f^{n}:=\operatorname{Pf}\left(t_{n}\right)$.

We may also construct a scheme that requires solving only linear systems of equations for each $n$ : Consider first the following Taylor formula for $v, w \in \mathbf{Z}$ and each pair of indexes $i, \alpha$ :

$$
\begin{aligned}
S_{i \alpha}(\nabla v)= & S_{i \alpha}(\nabla w)+\sum_{j, \beta=1}^{N} \partial_{\beta}\left(v_{j}-w_{j}\right) \frac{\partial S_{i \alpha}}{\partial \eta_{j \beta}}(\nabla w) \\
& +\sum_{j, \beta, k, \gamma=1}^{N} \partial_{\beta}\left(v_{j}-w_{j}\right) \partial_{\gamma}\left(v_{k}-w_{k}\right) \\
& \times \int_{0}^{1} \frac{\partial S_{i \alpha}}{\partial \eta_{j \beta} \partial \eta_{k \gamma}}(\nabla w+\tau \nabla(v-w)) d \tau .
\end{aligned}
$$

For a given function $y(t)$ defined on $[0, T]$ let

$$
\hat{y}^{n+1}=2 y^{n}-y^{n-1} \text {. }
$$

(Then for $y$ smooth enough, $\hat{y}^{n+1}-y^{n+1}=O\left(k^{2}\right)$.)

Now putting $v=u_{h}^{n+1}$ and $w=\hat{u}_{h}^{n+1}$ in (3.3), dropping the last term of the resulting equation (presumably of $O\left(k^{2}\right)$ ), and using (3.1), (1.4), and (3.4), we arrive at the following fully discrete scheme: Seek $U^{j} \in S_{h}, 0 \leq j \leq J$, approximating $u^{j}=u\left(t_{j}\right)$, by defining $U^{n+1} \in S_{h}$ as the solution of the linear system

$$
\begin{aligned}
\left(U^{n+1}\right. & \left.-2 U^{n}+U^{n-1}, \chi\right)+k^{2} q_{1} \tilde{a}\left(\widehat{U}^{n+1} ; U^{n+1}-\widehat{U}^{n+1}, \chi\right) \\
& +k^{2} q_{1} a\left(\widehat{U}^{n+1}, \chi\right)-2 k^{2} p_{1} a\left(U^{n}, \chi\right)+k^{2} q_{1} a\left(U^{n-1}, \chi\right) \\
= & k^{2}\left(q_{1} f^{n+1}-2 p_{1} f^{n}+q_{1} f^{n-1}, \chi\right), \quad \forall \chi \in S_{h},
\end{aligned}
$$

with $U^{0}, U^{1}$ given in $S_{h}$.

In the sequel we shall first analyze the error of the method (3.5) and show that, if the initial values $U^{0}, U^{1}$ are chosen so that

$$
\left\|E^{1}-E^{0}\right\|^{2}+k^{2}\left(\left\|E^{0}\right\|_{1}^{2}+\left\|E^{1}\right\|_{1}^{2}\right) \leq c k^{2}\left(k^{2}+h^{r}\right)^{2},
$$


where $E^{j}=U^{j}-W^{j}$, then

$$
\max _{0 \leq n \leq J}\left\|U^{n}-u\left(t_{n}\right)\right\| \leq c\left(h^{r}+k^{2}\right),
$$

under the assumptions $(\alpha),(\beta),(\gamma)$ of $\S 1$. A similar result, cf. Theorem 3.2, holds for the solutions $V^{n}$ of the nonlinear scheme (3.2).

For the error analysis we shall compare $U^{n}$ with the nonlinear elliptic projection $W^{n}$ of the solution $u\left(t_{n}\right)$ of $(1.1)$, defined by (1.5). We shall need the following consistency result, the proof of which can be found in the Appendix of [18]; see also [16].

Lemma 3.1. For $h$ sufficiently small and $1 \leq n \leq J-1$ there holds

$$
\begin{aligned}
\left(W^{n+1}\right. & \left.-2 W^{n}+W^{n-1}, \chi\right)+k^{2} q_{1} \tilde{a}\left(\widehat{W}^{n+1} ; W^{n+1}-\widehat{W}^{n+1}, \chi\right) \\
& +k^{2} q_{1} a\left(\widehat{W}^{n+1}, \chi\right)-2 k^{2} p_{1} a\left(W^{n}, \chi\right)+k^{2} q_{1} a\left(W^{n-1}, \chi\right) \\
= & -\left(\Lambda_{n}, \chi\right)+k^{2}\left(q_{1} f^{n+1}-2 p_{1} f^{n}+q_{1} f^{n-1}, \chi\right), \quad \forall \chi \in S_{h},
\end{aligned}
$$

where $\Lambda_{n}$ satisfies

$$
\left|\left(\Lambda_{n}, \chi\right)\right| \leq c k^{2}\left(k^{2}+k h^{r-1}+h^{r}\right)(\|\chi\|+k\|\nabla \chi\|), \quad \chi \in S_{h} .
$$

Now let $E^{n}=U^{n}-W^{n}$. Then, using (3.5) and Lemma 3.1, we obtain for $\chi \in S_{h}$

$$
\begin{aligned}
& \left(E^{n+1}-2 E^{n}+E^{n-1}, \chi\right) \\
& \quad+k^{2} q_{1}\left[\tilde{a}\left(\widehat{U}^{n+1} ; U^{n+1}-\widehat{U}^{n+1}, \chi\right)-\tilde{a}\left(\widehat{W}^{n+1} ; W^{n+1}-\widehat{W}^{n+1}, \chi\right)\right] \\
& \quad+k^{2} q_{1}\left[a\left(\widehat{U}^{n+1}, \chi\right)-a\left(\widehat{W}^{n+1}, \chi\right)\right]-2 k^{2} p_{1}\left[a\left(U^{n}, \chi\right)-a\left(W^{n}, \chi\right)\right] \\
& \quad+k^{2} q_{1}\left[a\left(U^{n-1}, \chi\right)-a\left(W^{n-1}, \chi\right)\right]=\left(\Lambda_{n}, \chi\right), \quad 1 \leq n \leq J-1 .
\end{aligned}
$$

For purposes of easy reference, we rewrite Taylor's formula (3.3) as

$$
a(\phi, \chi)-a(\psi, \chi)=\tilde{a}(\psi ; \phi-\psi, \chi)+b(\phi-\psi, \chi),
$$

where we have assumed that $\nabla \phi, \nabla \psi \in \mathbf{Z}, \phi, \psi \in S_{h}$, and where

$$
\begin{array}{r}
b(\phi-\psi, \chi)=\sum_{i, \alpha, j, \beta, k, \gamma=1}^{N}\left(\partial_{\beta}\left(\phi_{j}-\psi_{j}\right) \partial_{\gamma}\left(\phi_{k}-\psi_{k}\right)\right. \\
\left.\quad \times \int_{0}^{1} \frac{\partial S_{i \alpha}}{\partial \eta_{j \beta} \partial \eta_{k \gamma}}(\nabla \psi+\tau \nabla(\phi-\psi)) d \tau, \partial_{\alpha} \chi\right) .
\end{array}
$$

Using the fact that $S_{i \alpha}$ are smooth functions, we have that

$$
|b(\phi-\psi, \chi)| \leq c|\nabla(\phi-\psi)|_{\infty}\|\nabla(\phi-\psi)\|\|\nabla \chi\| .
$$

Let us assume that $\nabla U^{n+1}, \nabla \widehat{U}^{n+1} \in \mathbf{Z}$. Then it is easy to verify, using (3.8), that

$$
\begin{aligned}
{\left[\tilde { a } \left(\widehat{U}^{n+1}\right.\right.} & \left.\left.; U^{n+1}-\widehat{U}^{n+1}, \chi\right)-\tilde{a}\left(\widehat{W}^{n+1} ; W^{n+1}-\widehat{W}^{n+1}, \chi\right)\right] \\
& +\left[a\left(\widehat{U}^{n+1}, \chi\right)-a\left(\widehat{W}^{n+1}, \chi\right)\right] \\
= & \tilde{a}\left(\widehat{W}^{n+1} ; U^{n+1}-W^{n+1}, \chi\right) \\
& +\left[\tilde{a}\left(\widehat{U}^{n+1} ; U^{n+1}-\widehat{U}^{n+1}, \chi\right)-\tilde{a}\left(\widehat{W}^{n+1} ; U^{n+1}-\widehat{U}^{n+1}, \chi\right)\right] \\
& +b\left(\widehat{U}^{n+1}-\widehat{W}^{n+1}, \chi\right) .
\end{aligned}
$$


Starting from (3.7), using (3.10), and applying (3.8) for $\phi=U^{j}, \psi=W^{j}$, $j=n, n-1$, we finally come to the following error equation, which holds for all $\chi \in S_{h}$ if $\nabla U^{j} \in \mathbf{Z}, j=n, n-1, \nabla \hat{U}^{n+1} \in \mathbf{Z}$ :

$$
\begin{gathered}
\left(E^{n+1}-2 E^{n}+E^{n-1}, \chi\right)+k^{2} \tilde{a}\left(W^{n} ; q_{1} E^{n+1}-2 p_{1} E^{n}+q_{1} E^{n-1}, \chi\right) \\
=\left(B_{1}^{n}, \chi\right)+\left(B_{2}^{n}, \chi\right)+\left(B_{3}^{n}, \chi\right)+\left(\Lambda_{n}, \chi\right), \quad 1 \leq n \leq J-1,
\end{gathered}
$$

where

$$
\begin{aligned}
& B_{1}^{n}=-q_{1} k^{2}\left[\tilde{a}\left(\widehat{U}^{n+1} ; U^{n+1}-\widehat{U}^{n+1}, \chi\right)-\tilde{a}\left(\widehat{W}^{n+1} ; U^{n+1}-\widehat{U}^{n+1}, \chi\right)\right], \\
& B_{2}^{n}=-k^{2}\left[q_{1} b\left(\widehat{U}^{n+1}-\widehat{W}^{n+1}, \chi\right)-2 p_{1} b\left(U^{n}-W^{n}, \chi\right)+q_{1} b\left(U^{n-1}-W^{n-1}, \chi\right)\right], \\
& B_{3}^{n}=-q_{1} k^{2}\left\{\left[\tilde{a}\left(\widehat{W}^{n+1} ; E^{n+1}, \chi\right)-\tilde{a}\left(W^{n} ; E^{n+1}, \chi\right)\right]\right. \\
& \left.\quad+\left[\tilde{a}\left(W^{n-1} ; E^{n-1}, \chi\right)-\tilde{a}\left(W^{n} ; E^{n-1}, \chi\right)\right]\right\} .
\end{aligned}
$$

Choosing $\chi=E^{n+1}-E^{n-1}$ in (3.11), using the symmetry of $\tilde{a}(\phi ; \cdot, \cdot)$, $\nabla \phi \in \mathbf{Z}$, summing from $n=1$ to $M, 1 \leq n \leq M \leq J-1$, and estimating the $B_{i}^{n}$ terms, we can prove the following proposition, whose proof is also given in [18], [16].

Proposition 3.1. Assume that the initial values $U^{0}, U^{1} \in S_{h}$ are chosen so that

$$
\left\|E^{1}-E^{0}\right\|^{2}+k^{2}\left(\left\|\nabla E^{1}\right\|^{2}+\left\|\nabla E^{0}\right\|^{2}\right) \leq c k^{2}\left(k^{2}+h^{r}\right)^{2} .
$$

If $U^{n}, 0 \leq n \leq M+1 \leq J$, exist in $S_{h}$ and satisfy $\nabla U^{n} \in \mathbf{Z}, 0 \leq n \leq M$, and $\nabla \widehat{U}^{n+1} \in \mathbf{Z}, 1 \leq n \leq M$, then, there exists a positive constant $c$ independent of $h$ and $k$ such that

$$
\begin{aligned}
\mathscr{E}_{M+1} \leq & c M k\left(k^{2}+k h^{r-1}+h^{r}\right)^{2} k^{2} \\
& +c k \sum_{n=1}^{M}\left\{\left\|E^{n+1}-E^{n-1}\right\|^{2}+k^{2}\left(\left\|\nabla E^{n+1}\right\|^{2}+\left\|\nabla E^{n}\right\|^{2}+\left\|\nabla E^{n-1}\right\|^{2}\right)\right\} \\
& +c \sum_{n=1}^{M} k^{2}\left(\left|\nabla \widehat{E}^{n+1}\right|_{\infty}+\left|\nabla \widehat{E}^{n+1}\right|_{\infty}^{2}+\left|\nabla E^{n}\right|_{\infty}+\left|\nabla E^{n-1}\right|_{\infty}\right) \\
& \times\left(\left\|\nabla E^{n+1}\right\|^{2}+\left\|\nabla E^{n}\right\|^{2}+\left\|\nabla E^{n-1}\right\|^{2}\right), \quad 1 \leq n \leq M,
\end{aligned}
$$

where

$$
\begin{aligned}
\mathscr{C}_{n}=\left\|E^{n}-E^{n-1}\right\|^{2} & +k^{2} M_{0}\left(q_{1}+p_{1}\right) / 2\left\|\nabla\left(E^{n}-E^{n-1}\right)\right\|^{2} \\
& +k^{2} M_{0}\left(q_{1}-p_{1}\right) / 2\left\|\nabla\left(E^{n}+E^{n-1}\right)\right\|^{2}
\end{aligned}
$$

and $M_{0}$ is the constant appearing in $(\mathrm{S} 2 \mathrm{~b})$.

We are now ready to state and prove the convergence result for the scheme (3.5).

Theorem 3.1. Assume that the initial values $U^{0}, U^{1} \in S_{h}$ satisfy (3.12), and suppose that $k, h$ satisfy the mesh conditions $(\alpha),(\beta),(\gamma)$ of $\S 1$. Then there exists a positive constant $C$ independent of $h$ and $k$ such that

$$
\max _{0 \leq n \leq J}\left\|U^{n}-u\left(t_{n}\right)\right\| \leq C\left(h^{r}+k^{2}\right) .
$$

Proof. We observe that if the terms $\left|\nabla E^{j}\right|_{\infty}, j=1, \ldots, M$, in (3.13) can be bounded by $k$, then applying Gronwall's lemma, we are led to (3.15). In order 
to do this, we will use an induction argument. The induction hypothesis on $M$ is

(I) $U^{j} \in S_{h}, j=0,1, \ldots, M$, and $\nabla U^{j} \in \mathbf{Z}, j=0,1, \ldots, M$, and $\nabla \widehat{U}^{j+1} \in \mathbf{Z}, j=1,2, \ldots, M$;

(II) for $j=1, \ldots, M$ there holds $\left|\nabla E^{j}\right|_{\infty} \leq k$.

It is easy to see that (3.12) and (ii,b) give (I), (II) for $M=1$. Suppose now that (I) and (II) hold for some $M$, with $1 \leq M \leq J-1$. Then the hypotheses of Proposition 3.1 are fulfilled, and (3.13) gives

$$
\mathscr{E}_{M+1} \leq c k^{2}\left(k^{2}+k h^{r-1}+h^{r}\right)^{2}+c k \sum_{n=1}^{M+1} E_{n, n-1},
$$

where

$$
E_{n, n-1}=\left\|E^{n}-E^{n-1}\right\|^{2}+k^{2}\left\|\nabla\left(E^{n}+E^{n-1}\right)\right\|^{2}+k^{2}\left\|\nabla\left(E^{n}-E^{n-1}\right)\right\|^{2} .
$$

When $q_{1}>1 / 4$ it follows that $p_{1}+q_{1}>0$ and $q_{1}-p_{1}>0$. Hence, we can choose a $k_{0}$ such that for $k<k_{0}$ we have

$$
E_{M+1, M} \leq C_{*} k^{2}\left(k^{2}+k h^{r-1}+h^{r}\right)^{2}+C_{*} k \sum_{n=1}^{M} E_{n, n-1},
$$

where $C_{*}$ is a constant independent of $h, k$ but also independent of $M$. We shall follow this convention in the sequel as well; i.e., $C_{*}$ will denote a positive constant, not necessarily the same at any two places, but always independent of $h, k$, and $M$.

The discrete Gronwall lemma now gives

$$
\begin{aligned}
E_{n+1, n} & \leq C_{*} k^{2}\left(k^{2}+k h^{r-1}+h^{r}\right)^{2} \exp \left(C_{*} k n\right) \\
& \leq C_{*} k^{2}\left(k^{2}+k h^{r-1}+h^{r}\right)^{2}, \quad n=1,2, \ldots, M .
\end{aligned}
$$

Since $\left\|E^{n}\right\| \leq \sum_{j=1}^{n}\left\|E^{j}-E^{j-1}\right\|+\left\|E^{0}\right\|$, taking square roots on both sides of (3.16), and using (3.12) once more, we have

$$
\left\|E^{n}\right\| \leq C_{*}\left(k^{2}+k h^{r-1}+h^{r}\right), \quad n=0,1,2, \ldots, M+1 .
$$

Now (ii,b) and (3.16) give

$$
\left|\nabla E^{M+1}\right|_{\infty} \leq C_{*} h^{-N / 2}\left\|\nabla E^{M+1}\right\| \leq C_{*} h^{-N / 2}\left(k^{2}+k h^{r-1}+h^{r}\right)
$$

and

$$
\left|\nabla \widehat{E}^{M+2}\right|_{\infty} \leq C_{*} h^{-N / 2}\left(k^{2}+k h^{r-1}+h^{r}\right) .
$$

From our hypotheses we have $\left|\nabla E^{M+1}\right|_{\infty},\left|\nabla \widehat{E}^{M+2}\right|_{\infty} \leq \delta / 2$ for sufficiently small $h$ and $k$. Taking $h$ small enough so that $\left|W^{M+1}-u^{M+1}\right|_{1, \infty}$, $\left|\widehat{W}^{M+2}-\hat{u}^{M+2}\right|_{1, \infty} \leq \delta / 2$, we have $\nabla U^{M+1}, \nabla \widehat{U}^{M+2} \in \mathbf{Z}$, i.e., that (I) is valid for $M+1$.

For the proof of (II) we observe first that (3.16), (ii,b) give ( $C_{3}$ is the constant in (ii,b))

$$
\left|\nabla E^{M+1}\right|_{\infty} \leq C_{3} h^{-N / 2}\left\|\nabla E^{M+1}\right\| \leq C_{3} C_{*} h^{-N / 2}\left(k^{2}+k h^{r-1}+h^{r}\right),
$$

and we distinguish the following cases:

(1) When $N=1$, then from our assumptions it follows, for sufficiently small $k$ and $h$, that $\left|\nabla E^{M+1}\right|_{\infty} \leq k$, i.e., that (II) holds. 
(2) When $N=2$, the assumption $(\beta)$ of $\S 1$ yields

$$
3 C_{3} C_{*} k h^{-1} \leq 1, \quad 3 C_{3} C_{*} h^{-2} h^{r} \leq k h^{-1} \text { and } r>2,
$$

and therefore that $\left|\nabla E^{M+1}\right|_{\infty} \leq k$.

(3) When $N=3$, for $\left|\nabla E^{M+1}\right|_{\infty} \leq k$ to hold, it suffices that $3 C_{3} C_{*} k h^{-3 / 2} \leq$ 1 and $3 C_{3} C_{*} h^{r} h^{-3 / 2} \leq k$ or $3 C_{3} C_{*} h^{-3} h^{r} \leq k h^{-3 / 2} \leq\left(3 C_{3} C_{*}\right)^{-1}$. This relation is a consequence of assumption $(\gamma)$ of $\S 1$ for small $h$.

In conclusion, therefore, we have proved that (I), (II) hold for $M+1$, and thus (3.17) holds for any $M$ with $0 \leq M \leq J-1$; the proof of the theorem is complete.

Remark. A possible choice of the starting values $U^{0}, U^{1}$ is: Let $U^{0}=W^{0}$ and $U^{1}=W^{0}+k P u^{1}+k^{2} P u^{(2)}(0) / 2$, where $P$ is the $L^{2}$ projection on $S_{h}$, and $u^{(2)}(0)$ is computed using (1.1). This choice requires the solution of one nonlinear system. One can verify that the $U^{0}, U^{1}$ defined above satisfy (3.12).

A completely analogous result holds for the nonlinear scheme (3.2). In particular, we have the following theorem whose proof is given in [18], [16], and is carried out using Banach's fixed point theorem.

Theorem 3.2. We assume that the initial values $V^{0}, V^{1} \in S_{h}$ for the scheme (3.2) satisfy $\left(\varepsilon^{n}=V^{n}-W^{n}\right)$

$$
\left\|\varepsilon^{1}-\varepsilon^{0}\right\|^{2}+k^{2}\left(\left\|\nabla \varepsilon^{1}\right\|^{2}+\left\|\nabla \varepsilon^{0}\right\|^{2}\right) \leq c k^{2}\left(k^{2}+h^{r}\right)^{2} .
$$

If $k, h$ satisfy the mesh hypotheses $(\alpha),(\beta),(\gamma)$ of $\S 1$, then for every $n, 2 \leq$ $n \leq J$, the solution $V^{n}$ of (3.2) exists in $S_{h}$. Moreover, there exists a constant $C$ independent of $k, h$ such that

$$
\max _{0 \leq n \leq J}\left\|V^{n}-u\left(t_{n}\right)\right\| \leq C\left(h^{r}+k^{2}\right) .
$$

\section{HIGH-ORDER SINGLE-STEP FULLY DISCRETE SCHEMES}

In this section we shall construct high-order in time single-step fully discrete schemes to approximate the solution of (1.1). To motivate the construction, consider the semidiscrete approximation of $u, u_{h}:[0, T] \rightarrow S_{h}$, which satisfies (1.6) with given initial values $u_{h}(0)$ and $\dot{u}_{h}(0)$ in $S_{h}$. If

$$
\mathscr{U}_{h}(t)=\left(\begin{array}{l}
u_{h}(t) \\
\dot{u}_{h}(t)
\end{array}\right), \quad F=\left(\begin{array}{c}
0 \\
f_{h}
\end{array}\right),
$$

the semidiscrete equation can be written as a first-order system

$$
\left(\mathscr{U}_{h, t}, \Phi\right)-\left(\mathscr{A} \mathscr{U}_{h}, \Phi\right)=(F, \Phi), \quad \forall \Phi \in S_{h} \times S_{h},
$$

where $\mathscr{A}: S_{h} \times S_{h} \rightarrow S_{h} \times S_{h}$ is the operator defined by

$$
\mathscr{A}\left(\begin{array}{l}
\psi_{1} \\
\psi_{2}
\end{array}\right)=\left(\begin{array}{c}
\psi_{2} \\
-A \psi_{1}
\end{array}\right), \quad\left(\begin{array}{l}
\psi_{1} \\
\psi_{2}
\end{array}\right) \in S_{h} \times S_{h},
$$

and $A$ has been defined by $(1.3)$, and where by $(\cdot, \cdot)$ we denote the $L^{2} \times L^{2}$ inner product as well.

The fully discrete schemes which we are going to construct are based on up to fourth-order accurate rational approximations to the exponential. Following [1, 3,4 , we consider the rational function $\tilde{r}(z)=P(z) / Q(z)$, where $P$ and $Q$ are 
relatively prime polynomials of degree up to two, given by $P(z)=1+p_{1} z+p_{2} z^{2}$, $Q(z)=1+q_{1} z+q_{2} z^{2}$. We suppose that $\tilde{r}$ has the following properties of accuracy and stability: There exists an integer $\nu, 1 \leq \nu \leq 4$, such that

$$
\begin{gathered}
\left|\tilde{r}(z)-e^{z}\right| \leq c|z|^{\nu+1}, \quad|z| \rightarrow 0, \\
|\tilde{r}(z)| \leq 1 \quad \text { for } z \in i \mathbf{R} .
\end{gathered}
$$

An immediate consequence of (Rii) is that

$$
Q(z) \neq 0 \text { for } z \in i \mathbf{R} \text {. }
$$

It is easy to see now that for any smooth function $y=y(t)$ and $k>0$, there holds

$$
\begin{aligned}
& y(t+k)+q_{1} k y^{\prime}(t+k)+q_{2} k^{2} y^{\prime \prime}(t+k) \\
& \quad=y(t)+p_{1} k y^{\prime}(t)+p_{2} k^{2} y^{\prime \prime}(t)+O\left(k^{\nu+1} y^{(\nu+1)}\right) .
\end{aligned}
$$

Motivated by (4.2), we proceed to the construction of the scheme. The first derivative $\mathscr{U}_{h, t}$ is given by (4.1). For the approximation of the second derivative we have

$$
\left(\mathscr{U}_{h, t t}, \Phi\right)=\left(\left(\begin{array}{c}
\ddot{u}_{h} \\
-\partial_{t}\left(A u_{h}\right)
\end{array}\right), \Phi\right)+\left(\left(\begin{array}{l}
0 \\
\dot{f}
\end{array}\right), \Phi\right), \quad \Phi \in S_{h} \times S_{h} .
$$

The definition of $A$ yields for $\chi \in S_{h}$

$$
\begin{aligned}
\left(\partial_{t}\left(A u_{h}\right), \chi\right) & =\sum_{i, \alpha, j, \beta=1}^{N}\left(\frac{\partial S_{i \alpha}}{\partial \eta j \beta}\left(\nabla u_{h}\right) \partial_{\beta} \dot{u}_{h j}, \partial_{\alpha} \chi_{i}\right) \\
& =\tilde{a}\left(u_{h} ; \dot{u}_{h}, \chi\right)=\left(L\left(u_{h}\right) \dot{u}_{h}, \chi\right) .
\end{aligned}
$$

From the above relations we have for $\mathscr{U}^{n}:=\mathscr{U}_{h}\left(t_{n}\right), t_{n}=n k, k=0,1, \ldots, J$, $J k=T$,

(4.3) $\mathscr{U}^{n+1}+k q_{1} \mathscr{A} \mathscr{U}^{n+1}+k^{2} q_{2} \mathscr{A}^{\prime} \mathscr{U}^{n+1} \approx \mathscr{U}^{n}+k p_{1} \mathscr{A} \mathscr{U}^{n}+k^{2} p_{2} \mathscr{A}^{\prime} \mathscr{U}^{n}+F^{n}$

where we denote

$$
F^{n}=-\left(\begin{array}{c}
k^{2}\left(q_{2} f^{n+1}-p_{2} f^{n}\right) \\
k\left(q_{1} f^{n+1}-p_{1} f^{n}\right)+k^{2}\left(q_{2} f^{(1) n+1}-p_{2} f^{(1) n}\right)
\end{array}\right),
$$

$f^{n}:=P f\left(t_{n}\right), f^{(1) n}:=P \dot{f}\left(t_{n}\right)$, and $\mathscr{A}^{\prime}: S_{h} \times S_{h} \rightarrow S_{h} \times S_{h}$ is the operator defined by

$$
\mathscr{A}^{\prime}\left(\begin{array}{l}
\psi_{1} \\
\psi_{2}
\end{array}\right)=\left(\begin{array}{c}
-A \psi_{1} \\
-L\left(\psi_{1}\right) \psi_{2}
\end{array}\right), \quad\left(\begin{array}{l}
\psi_{1} \\
\psi_{2}
\end{array}\right) \in S_{h} \times S_{h} .
$$

Let $U_{1}^{n} \in S_{h}$ be the approximation of $u\left(t_{n}\right)$ which we shall compute from our scheme. Also let $\widehat{U}_{1}^{n+1}$ be a linear combination of previous values $U_{1}^{j}, n-m \leq$ $j \leq n$, which approximates $u\left(t_{n+1}\right)$; for the specific formula cf. (4.6) below. Starting from (4.3), putting $v=U_{1}^{n+1}$ and $w=\widehat{U}_{1}^{n+1}$ in the Taylor formula (3.3) and dropping second-order terms with respect to $\nabla\left(\widehat{U}_{1}^{n+1}-U_{1}^{n+1}\right)$, we finally obtain the following linear fully discrete scheme to find approximations $U^{n} \approx\left(\begin{array}{l}u\left(t_{n}\right) \\ \dot{u}\left(t_{n}\right)\end{array}\right), U^{n} \in S_{h} \times S_{h}$ : Let $U^{j} \in S_{h} \times S_{h}, 0 \leq j \leq n \leq J-1$, be given. 
Then define $U^{n+1} \in S_{h} \times S_{h}$ as the solution of the following linear system

$$
\begin{aligned}
U^{n+1} & +k q_{1} \widehat{\mathscr{L}}_{n+1} U^{n+1}+k^{2} q_{2} \widehat{\mathscr{L}}_{n+1}^{2} U^{n+1} \\
& -\left(\begin{array}{c}
k^{2} q_{2} A \widehat{U}_{1}^{n+1} \\
k q_{1} A \widehat{U}_{1}^{n+1}
\end{array}\right)+\left(\begin{array}{c}
k^{2} q_{2} L\left(\widehat{U}_{1}^{n+1}\right) \widehat{U}_{1}^{n+1} \\
k q_{1} L\left(\widehat{U}_{1}^{n+1}\right) \widehat{U}_{1}^{n+1}
\end{array}\right) \\
& -\left(U^{n}+k p_{1} \mathscr{A} U^{n}+k^{2} p_{2} \mathscr{A}^{\prime} U^{n}\right)=F^{n},
\end{aligned}
$$

where

$$
\widehat{\mathscr{L}}_{n+1}=\left(\begin{array}{cc}
O & I \\
-L\left(\widehat{U}_{1}^{n+1}\right) & O
\end{array}\right) .
$$

Our aim, now, is to show existence, uniqueness, and convergence of these approximations to the solution $u$ of (1.1) as $k, h \rightarrow 0$. In particular, we shall prove in this section that if $k^{-1} h^{-N / 2}\left(h^{r}+k^{\nu}\right)$ is small then, cf. Theorem 4.1 below,

$$
\max _{0 \leq n \leq J}\left(\left\|u\left(t_{n}\right)-U_{1}^{n}\right\|+\left\|\dot{u}\left(t_{n}\right)-U_{2}^{n}\right\|\right) \leq c\left(h^{r}+k^{\nu}\right) .
$$

We first introduce a family of norms to be used in the error estimations. If $w(t):=W(t)$ is the elliptic projection of the solution $u(t)$ of (1.1), defined by (1.5), we denote

$$
\mathscr{W}(t):=\left(\begin{array}{c}
w(t) \\
\dot{w}(t)
\end{array}\right) \in S_{h} \times S_{h}
$$

and $\mathscr{W}^{n}:=\mathscr{W}\left(t_{n}\right)$. We define now the following inner product in $S_{h} \times S_{h}$ : Suppose that $h$ is sufficiently small to ensure that $\nabla w^{n} \in \mathbf{Z}$, and let $\Phi=$ $\left(\begin{array}{l}\phi_{1} \\ \phi_{2}\end{array}\right), \quad \Psi=\left(\begin{array}{l}\psi_{1} \\ \psi_{2}\end{array}\right) \in S_{h} \times S_{h}$. Putting

$$
((\Phi, \Psi))_{n}=\left(L\left(w^{n}\right) \phi_{1}, \psi_{1}\right)+\left(\phi_{2}, \psi_{2}\right), \quad 0 \leq n \leq J,
$$

we observe, since $S_{h} \subset H_{0}^{1}$ and $L\left(w^{n}\right)$ satisfies (ii,a), that $((\cdot, \cdot))_{n}$ is an inner product on $S_{h} \times S_{h}$ with corresponding norm $\|\Phi\|_{n}^{2}:=\left(L\left(w^{n}\right) \phi_{1}, \phi_{1}\right)+$ $\left(\phi_{2}, \phi_{2}\right)=\left\|L^{1 / 2}\left(w^{n}\right) \phi_{1}\right\|^{2}+\left\|\phi_{2}\right\|^{2}, 0 \leq n \leq J$. Define now two operators of a form similar to $\widehat{\mathscr{L}}_{n}$ :

$$
\mathscr{L}_{n}=\left(\begin{array}{cc}
O & I \\
-L\left(w^{n}\right) & O
\end{array}\right) \quad \text { and } \quad \widetilde{\mathscr{L}}_{n+1}=\left(\begin{array}{cc}
O & I \\
-L\left(\hat{w}^{n+1}\right) & O
\end{array}\right) .
$$

We observe that $\left(\left(\mathscr{L}_{n} \Phi, \Phi\right)\right)_{n}=0, \forall \Phi \in S_{h} \times S_{h}$.

Consistency. Assume that for a smooth function $y=y(t)$, the approximation $\hat{y}^{n+1} \approx y^{n+1}$ is defined for $n \geq 3$ by

$$
\hat{y}^{n+1}=\alpha_{1} y^{n}+\alpha_{2} y^{n-1}+\alpha_{3} y^{n-2}+\alpha_{4} y^{n-3},
$$

where the numbers $\alpha_{1}, \alpha_{2}, \alpha_{3}, \alpha_{4}$ are chosen such that $\left|\hat{y}^{n+1}-y^{n+1}\right| \leq c k^{\nu}$ for every $n, 3 \leq n \leq J-1$. (Obviously, we may take $\alpha_{4}=0$ if $\nu=3$, $\alpha_{4}=\alpha_{3}=0$ if $\nu=2$, etc.)

We have now the following result.

Lemma 4.1. For $3 \leq n \leq J-1$ and $h$ sufficiently small, we have

$$
\begin{aligned}
\mathscr{W}^{n+1} & +k q_{1} \widetilde{\mathscr{L}}_{n+1} \mathscr{W}^{n+1}+k^{2} q_{2} \widetilde{\mathscr{L}}_{n+1}^{2} \mathscr{W}^{n+1} \\
& -\left(\begin{array}{c}
k^{2} q_{2} A \hat{w}^{n+1} \\
k q_{1} A \hat{w}^{n+1}
\end{array}\right)+\left(\begin{array}{c}
k^{2} q_{2} L\left(\hat{w}^{n+1}\right) \hat{w}^{n+1} \\
k q_{1} L\left(\hat{w}^{n+1}\right) \hat{w}^{n+1}
\end{array}\right) \\
& -\left(\mathscr{W}^{n}+k p_{1} \mathscr{A} \mathscr{W}^{n}+k^{2} p_{2} \mathscr{A}^{\prime} \mathscr{W}^{n}\right)=F^{n}-\Gamma_{n},
\end{aligned}
$$


where $\Gamma_{n} \in S_{h}$ satisfies

$$
\left\|\Gamma_{n}\right\|_{n} \leq c k^{\nu+1}\left(k^{2} h^{-1}+k^{2} h^{r-3}+k^{3} h^{-2}+1\right)+c k h^{r}\left(1+k h^{-1}\right) .
$$

Proof. For the terms of first order in $k$ we have, choosing $h$ small enough to ensure $\nabla w(t) \in \mathbf{Z}, 0 \leq t \leq T$, that

$$
\begin{gathered}
k q_{1} \widetilde{\mathscr{L}}_{n+1} \mathscr{W}^{n+1}-\left(\begin{array}{c}
0 \\
k q_{1} A \hat{w}^{n+1}
\end{array}\right)+\left(\begin{array}{c}
0 \\
k q_{1} L\left(\hat{w}^{n+1}\right) \hat{w}^{n+1}
\end{array}\right)-k p_{1} \mathscr{A} \mathscr{W}^{n} \\
=k q_{1} \mathscr{A} \mathscr{W}^{n+1}-k p_{1} \mathscr{A} \mathscr{W}^{n}+\left(\begin{array}{c}
0 \\
k q_{1} B\left(w^{n+1}, \hat{w}^{n+1}\right)
\end{array}\right)
\end{gathered}
$$

where the operator $B: S_{h} \times S_{h} \rightarrow S_{h}$ is defined as follows: For every $\chi \in S_{h}$, (4.8)

$$
\begin{aligned}
(B(\phi, \psi), \chi)=\sum_{i, \alpha, j, \beta, k, \gamma=1}^{N} & \left(\partial_{\beta}\left(\phi_{j}-\psi_{j}\right) \partial_{\gamma}\left(\phi_{k}-\psi_{k}\right)\right. \\
& \left.\times \int_{0}^{1} \frac{\partial S_{i \alpha}}{\partial \eta_{j \beta} \partial \eta_{k \gamma}}(\nabla \psi+\tau \nabla(\phi-\psi)) d \tau, \partial_{\alpha} \chi_{i}\right) .
\end{aligned}
$$

For the estimation of $B\left(w^{n+1}, \hat{w}^{n+1}\right)$ we use (v), (iv,a,b), (4.6), and the smoothness of $S_{i \alpha}$, so that

$$
\begin{aligned}
& \left|\left(B\left(w^{n+1}, \hat{w}^{n+1}\right), \chi\right)\right| \\
& \leq \mid \sum_{i, \alpha, j, \beta, k, \gamma=1}^{N}\left(\partial_{\beta}\left[\left(w_{j}^{n+1}-u_{j}^{n+1}\right)-\left(\hat{w}_{j}^{n+1}-\hat{u}_{j}^{n+1}\right)\right]\right. \\
& \left.\quad \times \partial_{\gamma}\left(w_{k}^{n+1}-\hat{w}_{k}^{n+1}\right) I_{i}^{n}, \partial_{\alpha} \chi_{i}\right) \mid \\
& +\mid \sum_{i, \alpha, j, \beta, k, \gamma=1}^{N}\left(\partial_{\beta}\left(u_{j}^{n+1}-\hat{u}_{j}^{n+1}\right)\right. \\
& \quad+\left|\sum_{i, \alpha, j, \beta, k, \gamma=1}^{N}\left(\partial_{\beta}\left(u_{j}^{n+1}-\hat{u}_{j}^{n+1}\right) \partial_{\gamma}\left(u_{k}^{n+1}-\hat{u}_{k}^{n+1}\right) I_{i}^{n}, \partial_{\alpha} \chi_{i}\right)\right| \\
& \left.\left.\left.\leq c k^{\nu+1} h^{r-1}\|\nabla \chi\|+c k^{\nu+2} \| \nabla \chi \chi_{k}^{n+1}-u_{k}^{n+1}\right)-\left(\hat{w}_{k}^{n+1}-\hat{u}_{k}^{n+1}\right)\right] I_{i}^{n}, \partial_{\alpha} \chi_{i}\right) \mid
\end{aligned}
$$

where

$I_{\mathbf{i}}^{n}:=\int_{0}^{1} \frac{\partial S_{i \alpha}}{\partial \eta_{j \beta} \partial \eta_{k \gamma}}\left(\nabla \hat{w}^{n+1}+\tau \nabla\left(w^{n+1}-\hat{w}^{n+1}\right)\right) d \tau \quad$ and $\quad \mathbf{i}:=(i, \alpha, j, \beta, k, \gamma)$.

Using (ii,a), we conclude

$$
k\left\|B\left(w^{n+1}, \hat{w}^{n+1}\right)\right\| \leq c k^{\nu+1}\left(k h^{r-2}+k^{2} h^{-1}\right) .
$$


In order to complete the estimation of the right-hand side of (4.7), we just substitute $k q_{1} \mathscr{A} \mathscr{W}^{n+1}-k p_{1} \mathscr{A} \mathscr{W}^{n}$ by

$$
\begin{aligned}
k q_{1} \mathscr{A} & \mathscr{W}^{n+1}-k p_{1} \mathscr{A} \mathscr{W}^{n} \\
& =\left(\begin{array}{c}
k q_{1} \dot{w}^{n+1}-k p_{1} \dot{w}^{n} \\
k q_{1} P \ddot{u}^{n+1}-k p_{1} P \ddot{u}^{n}
\end{array}\right)-\left(\begin{array}{c}
0 \\
k q_{1} f^{n+1}-k p_{1} f^{n}
\end{array}\right),
\end{aligned}
$$

where we have used that $A w^{n}=A u^{n}$ and (1.1) $\left(A u^{n}=f^{n}-P \ddot{u}^{n}\right)$.

For the estimation of the terms with coefficient $k^{2}$, we observe, using (3.3) and (4.8), that

$$
\begin{aligned}
k^{2} q_{2} & \widetilde{\mathscr{L}}_{n+1}^{2} \mathscr{W}^{n+1}-\left(\begin{array}{c}
k^{2} q_{2} A \hat{w}^{n+1}-k^{2} q_{2} L\left(\hat{w}^{n+1}\right) \hat{w}^{n+1} \\
0
\end{array}\right)-k^{2} p_{2} \mathscr{A}^{\prime} \mathscr{W}^{n} \\
= & k^{2} q_{2} \mathscr{A}^{\prime} \mathscr{W}^{n+1}-k^{2} p_{2} \mathscr{A}^{\prime} \mathscr{W}^{n}+\left(\begin{array}{c}
k^{2} q_{2} B\left(w^{n+1}, \hat{w}^{n+1}\right) \\
k^{2} q_{2}\left[L\left(w^{n+1}\right)-L\left(\hat{w}^{n+1}\right)\right] \dot{w}^{n+1}
\end{array}\right) .
\end{aligned}
$$

From (ii,a), (4.9) we have

$$
k^{2}\left\|L^{1 / 2}\left(w^{n+1}\right) B\left(w^{n+1}, \hat{w}^{n+1}\right)\right\| \leq c k^{\nu+1}\left(k^{2} h^{r-3}+k^{3} h^{-2}\right) .
$$

Also, the definition of $L(\cdot)$ and relations (4.6), (iv,b), (v) give

$$
\begin{aligned}
& \left|\left(\left[L\left(w^{n+1}\right)-L\left(\hat{w}^{n+1}\right)\right] \dot{w}^{n+1}, \chi\right)\right| \leq c\left\|w^{n+1}-\hat{w}^{n+1}\right\|_{1}\left|\dot{w}^{n+1}\right|_{1, \infty}\|\nabla \chi\| \\
& \quad \leq c\left(\left\|\left(w^{n+1}-u^{n+1}\right)-\left(\hat{w}^{n+1}-\hat{u}^{n+1}\right)\right\|_{1}+\left\|u^{n+1}-\hat{u}^{n+1}\right\|_{1}\right)\|\nabla \chi\| \\
& \quad \leq c\left(k^{\nu} h^{r-1}+k^{\nu}\right)\|\nabla \chi\| .
\end{aligned}
$$

Hence, using (ii,a), we obtain

$$
k^{2}\left\|\left[L\left(w^{n+1}\right)-L\left(\hat{w}^{n+1}\right)\right] \dot{w}^{n+1}\right\| \leq c k^{\nu+1}\left(k h^{-1}\right) .
$$

For the other terms of (4.11) we observe that differentiating $A w=A u$ with respect to $t$ and using (1.1), we obtain

$$
\begin{aligned}
k^{2} q_{2} \mathscr{A}^{\prime} \mathscr{W}^{n+1}-k^{2} p_{2} \mathscr{A}^{\prime} \mathscr{W}^{n}=\left(\begin{array}{c}
-k^{2} q_{2} A w^{n+1}+k^{2} p_{2} A w^{n} \\
-k^{2} q_{2} L\left(w^{n+1}\right) \dot{w}^{n+1}+k^{2} p_{2} L\left(w^{n}\right) \dot{w}^{n}
\end{array}\right) \\
=\left(\begin{array}{c}
k^{2} q_{2} P\left(\ddot{u}^{n+1}-\ddot{w}^{n+1}\right)-k^{2} p_{2} P\left(\ddot{u}^{n}-\ddot{w}^{n}\right) \\
0
\end{array}\right) \\
+\left(\begin{array}{c}
k^{2} q_{2} \ddot{w}^{n+1}-k^{2} p_{2} \ddot{w}^{n} \\
k^{2} q_{2} P u^{(3) n+1}-k^{2} p_{2} P u^{(3) n}
\end{array}\right)-\left(\begin{array}{c}
k^{2}\left(q_{2} f^{n+1}-p_{2} f^{n}\right) \\
k^{2}\left(q_{2} f^{(1) n+1}-p_{2} f^{(1) n}\right)
\end{array}\right) .
\end{aligned}
$$

Using (ii,a), (iv,b), we have

$$
k^{2}\left\|L^{1 / 2}\left(w^{n+1}\right)\left[P\left(\ddot{u}^{n+1}-\ddot{w}^{n+1}\right)\right]\right\| \leq c k h^{r}\left(k h^{-1}\right) .
$$

Note also that

$$
\mathscr{W}^{n+1}-\mathscr{W}^{n}=\left(\begin{array}{c}
w^{n+1}-w^{n} \\
P\left(\dot{u}^{n+1}-\dot{u}^{n}\right)
\end{array}\right)+\left(\begin{array}{c}
0 \\
P\left[\left(\dot{w}^{n+1}-\dot{u}^{n+1}\right)-\left(\dot{w}^{n}-\dot{u}^{n}\right)\right]
\end{array}\right)
$$

where (iv,b) again gives

$$
\left\|P\left[\left(\dot{w}^{n+1}-\dot{u}^{n+1}\right)-\left(\dot{w}^{n}-\dot{u}^{n}\right)\right]\right\| \leq c k h^{r} .
$$


Summarizing, we need finally an estimate for

$$
\begin{aligned}
\Gamma_{1}^{n}= & \left(\begin{array}{c}
w^{n+1}-w^{n} \\
P\left(\dot{u}^{n+1}-\dot{u}^{n}\right)
\end{array}\right)+k\left(\begin{array}{c}
q_{1} \dot{w}^{n+1}-p_{1} \dot{w}^{n} \\
q_{1} P\left[\ddot{u}^{n+1}-p_{1} \ddot{u}^{n}\right]
\end{array}\right) \\
& +k^{2}\left(\begin{array}{c}
q_{2} \ddot{w}-p_{2} \ddot{w}^{n} \\
q_{2} P u^{(3) n+1}-p_{2} P u^{(3) n}
\end{array}\right) .
\end{aligned}
$$

Using (4.2), (ii,a) and (iv,b), we obtain, putting $\rho(t)=w(t)-u(t)$,

$$
\begin{aligned}
& \left\|L^{1 / 2}\left(w^{n+1}\right)\left[\left(w^{n+1}+k q_{1} \dot{w}^{n+1}+k^{2} q_{2} \ddot{w}^{n+1}\right)-\left(w^{n}+k p_{1} \dot{w}^{n}+k^{2} p_{2} \ddot{w}^{n}\right)\right]\right\| \\
& \leq c\left\|\left(u^{n+1}+k q_{1} \dot{u}^{n+1}+k^{2} q_{2} \ddot{u}^{n+1}\right)-\left(u^{n}+k p_{1} \dot{u}^{n}+k^{2} p_{2} \ddot{u}^{n}\right)\right\|_{1} \\
& \quad+c\left\|\left(\rho^{n+1}+k q_{1} \dot{\rho}^{n+1}+k^{2} q_{2} \ddot{\rho}^{n+1}\right)-\left(\rho^{n}+k p_{1} \dot{\rho}^{n}+k^{2} p_{2} \ddot{\rho}^{n}\right)\right\|_{1} \\
& \leq c k^{\nu+1}\left(1+h^{r-1}\right) .
\end{aligned}
$$

Also, from (4.2) we have

$$
\left\|P\left[\left(\dot{u}^{n+1}+k q_{1} \ddot{u}^{n+1}+k^{2} q_{2} u^{(3) n+1}\right)-\left(\dot{u}^{n}+k p_{1} \ddot{u}^{n}+k^{2} p_{2} u^{(3) n}\right)\right]\right\| \leq c k^{\nu+1} .
$$

Therefore, we obtain

$$
\left\|\Gamma_{1}^{n}\right\|_{n+1} \leq c k^{\nu+1} .
$$

Now combining (4.7)-(4.19), we obtain the desired result.

The basic error equation. Let $E^{n}=U^{n}-\mathscr{W}^{n}$. Using the fully discrete scheme (4.5) and Lemma 4.1, we have for $3 \leq n \leq J-1$,

$$
\begin{aligned}
E^{n+1}+ & k q_{1}\left(\widehat{\mathscr{L}}_{n+1} U^{n+1}-\widetilde{\mathscr{L}}_{n+1} \mathscr{W}^{n+1}\right)+k^{2} q_{2}\left(\widehat{\mathscr{L}}_{n+1}^{2} U^{n+1}-\widetilde{\mathscr{L}}_{n+1}^{2} \mathscr{W}^{n+1}\right) \\
& -\left(\begin{array}{c}
k^{2} q_{2}\left[\left(A \widehat{U}_{1}^{n+1}-L\left(\widehat{U}_{1}^{n+1}\right) \widehat{U}_{1}^{n+1}\right)-\left(A \hat{w}^{n+1}-L\left(\hat{w}^{n+1}\right) \hat{w}^{n+1}\right)\right] \\
k q_{1}\left[\left(A \widehat{U}_{1}^{n+1}-L\left(\widehat{U}_{1}^{n+1}\right) \widehat{U}_{1}^{n+1}\right)-\left(A \hat{w}^{n+1}-L\left(\hat{w}^{n+1}\right) \hat{w}^{n+1}\right)\right]
\end{array}\right) \\
= & E^{n}+k p_{1}\left(\mathscr{A} U^{n}-\mathscr{A} \mathscr{W}^{n}\right)+k^{2} p_{2}\left(\mathscr{A}^{\prime} U^{n}-\mathscr{A}^{\prime} \mathscr{W}^{n}\right)+\Gamma^{n} .
\end{aligned}
$$

In the sequel we assume that $\nabla U_{1}^{n}, \nabla \widehat{U}_{1}^{n+1} \in \mathbf{Z}$. Then it is easy to verify that

$$
\begin{aligned}
-\left(L\left(\widehat{U}_{1}^{n+1}\right) U_{1}^{n+1}-L\left(\hat{w}^{n+1}\right) w^{n+1}\right)-\left(A \widehat{U}_{1}^{n+1}-A \hat{w}^{n+1}\right) \\
\quad+\left(L\left(\widehat{U}_{1}^{n+1}\right) \widehat{U}_{1}^{n+1}-L\left(\hat{w}^{n+1}\right) \hat{w}^{n+1}\right) \\
=-L\left(\hat{w}^{n+1}\right)\left(U_{1}^{n+1}-w^{n+1}\right)-B\left(\widehat{U}_{1}^{n+1}, \hat{w}^{n+1}\right) \\
\quad-\left[L\left(\widehat{U}_{1}^{n+1}\right)\left(U_{1}^{n+1}-\widehat{U}_{1}^{n+1}\right)-L\left(\hat{w}^{n+1}\right)\left(U_{1}^{n+1}-\widehat{U}_{1}^{n+1}\right)\right]
\end{aligned}
$$

and

$$
\begin{aligned}
L\left(\widehat{U}_{1}^{n+1}\right) U_{2}^{n+1}-L\left(\hat{w}^{n+1}\right) \dot{w}^{n+1}= & L\left(\hat{w}^{n+1}\right)\left(U_{2}^{n+1}-\dot{w}^{n+1}\right) \\
& +\left(L\left(\widehat{U}_{1}^{n+1}\right)-L\left(\hat{w}^{n+1}\right)\right) U_{2}^{n+1} .
\end{aligned}
$$

Noting also that $A U_{1}^{n}-A w^{n}=L\left(w^{n}\right)\left(U_{1}^{n}-w^{n}\right)+B\left(U_{1}^{n}, w^{n}\right)$ and $L\left(U_{1}^{n}\right) U_{2}^{n}-$ $L\left(w^{n}\right) \dot{w}^{n}=L\left(w^{n}\right)\left(U_{2}^{n}-\dot{w}^{n}\right)+\left(L\left(U_{1}^{n}\right)-L\left(w^{n}\right)\right) U_{2}^{n}$, we come to the basic error 
equation: If $\nabla U_{1}^{n}, \nabla \hat{U}_{1}^{n+1} \in \mathbf{Z}$, then there holds (4.20)

$$
\begin{aligned}
& E^{n+1}+k q_{1} \widetilde{\mathscr{L}}_{n+1} E^{n+1}+k^{2} q_{2} \widetilde{\mathscr{L}}_{n+1}^{2} E^{n+1} \\
& =E^{n}+k p_{1} \mathscr{L}_{n} E^{n}+k^{2} p_{2} \mathscr{L}_{n}^{2} E^{n} \\
& +\left(\begin{array}{c}
k^{2} q_{2}\left[L\left(\widehat{U}_{1}^{n+1}\right)\left(U_{1}^{n+1}-\widehat{U}_{1}^{n+1}\right)-L\left(\hat{w}^{n+1}\right)\left(U_{1}^{n+1}-\widehat{U}_{1}^{n+1}\right)\right] \\
k q_{1}\left[L\left(\widehat{U}_{1}^{n+1}\right)\left(U_{1}^{n+1}-\widehat{U}_{1}^{n+1}\right)-L\left(\hat{w}^{n+1}\right)\left(U_{1}^{n+1}-\widehat{U}_{1}^{n+1}\right)\right]
\end{array}\right) \\
& +\left(\begin{array}{c}
k^{2}\left[q_{2} B\left(\widehat{U}_{1}^{n+1}, \hat{w}^{n+1}\right)-p_{2} B\left(U_{1}^{n}, w^{n}\right)\right] \\
k\left[q_{1} B\left(\widehat{U}_{1}^{n+1}, \hat{w}^{n+1}\right)-p_{1} B\left(U_{1}^{n}, w^{n}\right)\right]
\end{array}\right) \\
& +\left(\begin{array}{c}
0 \\
\left.k^{2}\left[q_{2}\left(L\left(\widehat{U}_{1}^{n+1}\right)-L\left(\hat{w}^{n+1}\right)\right) U_{2}^{n+1}-p_{2}\left(L\left(U_{1}^{n}\right)-L\left(w^{n}\right)\right) U_{2}^{n}\right]+\Gamma^{n}\right)
\end{array}\right. \\
& =: E^{n}+k p_{1} \mathscr{L}_{n} E^{n}+k^{2} p_{2} \mathscr{L}_{n}^{2} E^{n}+\Lambda_{1}^{n}+\Lambda_{2}^{n}+\Lambda_{3}^{n}+\Gamma^{n}, \quad 3 \leq n \leq J-1 .
\end{aligned}
$$

Now letting

$$
\widetilde{Q}_{n+1}=Q\left(k \widetilde{\mathscr{L}}_{n+1}\right), \quad Q_{n}=Q\left(k \mathscr{L}_{n}\right), \quad \text { and } \quad P_{n}=P\left(k \mathscr{L}_{n}\right),
$$

we can give the error equation (4.20) the form

$$
\widetilde{Q}_{n+1} E^{n+1}=P_{n} E^{n}+\Lambda_{1}^{n}+\Lambda_{2}^{n}+\Lambda_{3}^{n}+\Gamma^{n}, \quad 3 \leq n \leq J-1 .
$$

Properties of the operators $\tilde{Q}_{n}, Q_{n}, P_{n}$. In what follows we shall show some properties of the operators occurring above. First note that

$$
\|\Phi\|_{n+1} \leq(1+c k)\|\Phi\|_{n}, \quad \forall \Phi \in S_{h} \times S_{h} .
$$

Indeed, from the definition of $\|\cdot\|_{n+1}$ we have $\|\Phi\|_{n+1}^{2}=\left(L\left(w^{n+1}\right) \phi_{1}, \phi_{1}\right)+$ $\left(\phi_{2}, \phi_{2}\right)$; hence (v), (ii,a), (S2b), and the definition of $L(v)$ give

$$
\begin{aligned}
& \|\Phi\|_{n+1}^{2}-\|\Phi\|_{n}^{2}=\left(\left[L\left(w^{n+1}\right)-L\left(w^{n}\right)\right] \phi_{1}, \phi_{1}\right) \\
& \quad \leq c k \sup _{s \in\left[t_{n-1}, t_{n+1}\right]}|\dot{w}(s)|_{1, \infty}\left\|\nabla \phi_{1}\right\|^{2} \leq c k\left(L\left(w^{n}\right) \phi_{1}, \phi_{1}\right) \leq c k\|\Phi\|_{n}^{2},
\end{aligned}
$$

or $\|\Phi\|_{n+1}^{2} \leq(1+c k)\|\Phi\|_{n}^{2} \leq(1+c k / 2)^{2}\|\Phi\|_{n}^{2}$, which gives (I).

An immediate consequence of (I), of the fact that $\mathscr{L}_{n}$ has purely imaginary eigenvalues, and of the stability assumption on $\tilde{r}$ is that

$$
\left\|P_{n} \Phi\right\|_{n+1} \leq(1+c k)\left\|Q_{n} \Phi\right\|_{n}, \quad \forall \Phi \in S_{h} \times S_{h} .
$$

We also need an estimate for $\left\|\left(Q_{n+1}-\widetilde{Q}_{n+1}\right) \Phi\right\|_{n+1}$. For this, first observe that

$$
\begin{aligned}
\left(Q_{n+1}-\tilde{Q}_{n+1}\right) \Phi= & k q_{1}\left(\begin{array}{c}
0 \\
{\left[L\left(\hat{w}^{n+1}\right)-L\left(w^{n+1}\right)\right] \phi_{1}}
\end{array}\right) \\
& +k^{2} q_{2}\left(\begin{array}{c}
{\left[L\left(\hat{w}^{n+1}\right)-L\left(w^{n+1}\right)\right] \phi_{1}} \\
{\left[L\left(\hat{w}^{n+1}\right)-L\left(w^{n+1}\right)\right] \phi_{2}}
\end{array}\right) .
\end{aligned}
$$

Relations (v), (ii,a) give

$$
\begin{aligned}
\left|\left(\left[L\left(\hat{w}^{n+1}\right)-L\left(w^{n+1}\right)\right] \phi_{1}, \chi\right)\right| & \leq c k \sup _{s \in\left[t_{n-1}, t_{n+1}\right]}|\dot{w}(s)|_{1, \infty}\left\|\nabla \phi_{1}\right\|\|\nabla \chi\| \\
& \leq c k h^{-1}\left(L\left(w^{n+1}\right) \phi_{1}, \phi_{1}\right)^{1 / 2}\|\chi\|
\end{aligned}
$$


and $k^{2}\left|\left(\left[L\left(\hat{w}^{n+1}\right)-L\left(w^{n+1}\right)\right] \phi_{2}, \chi\right)\right| \leq c k\left(k^{2} h^{-2}\right)\left\|\phi_{2}\right\|\|\chi\|$. Similarly, using (ii,a), (v),

$$
\begin{aligned}
& k^{2}\left|\left(L^{1 / 2}\left(w^{n+1}\right)\left[L\left(\hat{w}^{n+1}\right)-L\left(w^{n+1}\right)\right] \phi_{1}, \chi\right)\right| \\
& \quad \leq c k^{3} \sup _{s \in\left[t_{n-1}, t_{n+1}\right]}|\dot{w}(s)|_{1, \infty}\left\|\nabla \phi_{1}\right\|\left\|\nabla L^{1 / 2}\left(w^{n+1}\right) \chi\right\| \\
& \quad \leq c k\left(k^{2} h^{-2}\right)\left(L\left(w^{n+1}\right) \phi_{1}, \phi_{1}\right)^{1 / 2}\|\chi\| .
\end{aligned}
$$

Since now $\left(\left(\mathscr{L}_{n} \Phi, \Phi\right)\right)_{n}=0, \forall \Phi \in S_{h} \times S_{h}$, we have for any $\Phi \in S_{h} \times S_{h}$

$$
\begin{aligned}
\left\|Q_{n+1} \Phi\right\|_{n+1}^{2}= & \left\|L^{1 / 2}\left(w^{n+1}\right) \phi_{1}\right\|^{2}+\left(q_{1}^{2}-2 q_{2}\right) k^{2}\left\|L\left(w^{n+1}\right) \phi_{1}\right\|^{2} \\
& +q_{2}^{4} k^{4}\left\|L^{3 / 2}\left(w^{n+1}\right) \phi_{1}\right\|^{2}+\left\|\phi_{2}\right\|^{2}+\left(q_{1}^{2}-2 q_{2}\right) k^{2}\left\|L^{1 / 2}\left(w^{n+1}\right) \phi_{2}\right\|^{2} \\
& +q_{2}^{4} k^{4}\left\|L\left(w^{n+1}\right) \phi_{2}\right\|^{2} .
\end{aligned}
$$

Note that if $\nu \geq 3$, then (4.2) implies that $q_{2}=\frac{1}{2} q_{1}-\frac{1}{6}$. Hence, a straightforward computation shows that if $q_{2}=0$ for $\nu=2$ (no real restriction), then $q_{1}^{2}-2 q_{2}>0$ for $\nu=2,3,4$. Therefore, we have

$$
\left\|Q_{n+1} \Phi\right\|_{n+1}^{2} \geq\|\Phi\|_{n+1}^{2}, \quad \forall \Phi \in S_{h} \times S_{h} .
$$

Consequently, we have proved the relation

$$
\text { (IV) }\left\|\left(Q_{n+1}-\tilde{Q}_{n+1}\right) \Phi\right\|_{n+1} \leq c k\left(k h^{-1}+k^{2} h^{-2}\right)\left\|Q_{n+1} \Phi\right\|_{n+1}, \quad \forall \Phi \in S_{h} \times S_{h}
$$

Now using (4.21) and (I)-(IV), we finally obtain

$$
\begin{aligned}
\left\|Q_{n+1} E^{n+1}\right\|_{n+1}= & \left\|P_{n} E^{n}\right\|_{n+1}+\left\|\left(Q_{n+1}-\tilde{Q}_{n+1}\right) E^{n+1}\right\|_{n+1} \\
& +\left\|\Lambda_{1}^{n}+\Lambda_{2}^{n}+\Lambda_{3}^{n}+\Gamma^{n}\right\|_{n+1} \\
\leq & (1+c k)\left\|Q_{n} E^{n}\right\|_{n}+c k\left(k h^{-1}+k^{2} h^{-2}\right)\left\|Q_{n+1} E^{n+1}\right\|_{n+1} \\
& +\left\|\Lambda_{1}^{n}+\Lambda_{2}^{n}+\Lambda_{3}^{n}+\Gamma^{n}\right\|_{n+1}, \quad 3 \leq n \leq J-1 .
\end{aligned}
$$

Convergence. At this point, to complete the first phase of the error estimations, we need estimates for $\left\|\Lambda_{i}^{n}\right\|_{n+1}, i=1,2,3$. This is done in the following proposition, the proof of which can be found in [18], [16].

Proposition 4.1. Assume $U^{n}, U^{n+1}, \widehat{U}^{n+1} \in S_{h} \times S_{h}$ and $\nabla U_{1}^{n}, \nabla \widehat{U}_{1}^{n+1} \in \mathbf{Z}$. Then for $3 \leq n \leq J-1$ there holds

$$
\begin{aligned}
& \left\|Q_{n+1} E^{n+1}\right\|_{n+1} \leq(1+c k)\left\|Q_{n} E^{n}\right\|_{n} \\
& +c k\left\{k h^{-1}+k^{2} h^{-2}+\left(h^{-1}+k h^{-2}\right)\left|\widehat{E}_{1}^{n+1}\right|_{1, \infty}\right\}\left\|Q_{n+1} E^{n+1}\right\|_{n+1} \\
& +c k\left\{k h^{-1}+k^{2} h^{-2}+\left(h^{-1}+k h^{-2}\right)\left(\left|\widehat{E}_{1}^{n+1}\right|_{1, \infty}+\left|E_{n}^{n}\right|_{1, \infty}\right)\right. \\
& \quad \times\left(\left\|Q_{n} E^{n}\right\|_{n}+\left\|Q_{n-1} E^{n-1}\right\|_{n-1}+\left\|Q_{n-2} E^{n-2}\right\|_{n-2}\right. \\
& \left.\left.+\left\|Q_{n-3} E^{n-3}\right\|_{n-3}\right)\right\} \\
& +c k^{\nu+1}\left(1+k h^{-1}+k^{2} h^{r-3}+k^{3} h^{-2}\right)+c k h^{r}\left(1+k h^{-1}\right) . \quad
\end{aligned}
$$

We are now ready to state and prove the following convergence result.

Theorem 4.1. We assume that $U^{j} \in S_{h} \times S_{h}, j=0,1,2,3$, have been chosen in such a way as to satisfy $\left\|Q_{j} E^{j}\right\|_{j} \leq c\left(k^{\nu}+h^{r}\right)$. Then for every $n, 4 \leq n \leq J$, the element $U^{n} \in S_{h} \times S_{h}$ exists uniquely as the solution of the linear system (4.5). Let $k h^{-1}<\alpha$ for some $\alpha>0$ and $k^{-1} h^{-N / 2}\left(h^{r}+k^{\nu}\right) \leq \widehat{C}$, where $\widehat{C}$ is 
a sufficiently small constant and suppose that the rational function $\tilde{r}(x)$ satisfies (Ri), (Rii). Then, there exists a positive constant $c$ independent of $k, h$ such that

$$
\max _{0 \leq n \leq J}\left\|Q_{n} E^{n}\right\|_{n} \leq c\left(k^{\nu}+h^{r}\right),
$$

and consequently

$$
\max _{0 \leq n \leq J}\left(\left\|u^{n}-U_{1}^{n}\right\|+\left\|\dot{u}^{n}-U_{2}^{n}\right\|\right) \leq c\left(k^{\nu}+h^{r}\right) .
$$

Proof. We shall show the desired result by induction on $M, 0 \leq M \leq J-1$. The induction hypothesis on $M$ is

(a) $U^{j}$ exist in $S_{h} \times S_{h}, j=0,1, \ldots, M$, and $\nabla U_{1}^{j} \in \mathbf{Z}, j=0,1, \ldots, M$, and $\nabla \widehat{U}_{1}^{j+1} \in \mathbf{Z}, j=1,2, \ldots, M+1$;

(b) for $j=1, \ldots, M$ there holds $\left|\nabla E_{1}^{j}\right|_{\infty} \leq k$.

Since $\left\|Q_{j} E^{j}\right\|_{j} \leq c\left(k^{\nu}+h^{r}\right), j=0,1,2,3$, we have the validity of (a) for $M=3$. Also, using (ii,b), we see that $\left|E_{1}^{j}\right|_{1, \infty} \leq c h^{-N / 2}\left(h^{r}+k^{\nu}\right), j=0, \ldots, 3$, which gives (b) for $M=3$, taking $k^{-1} h^{-N / 2}\left(h^{r}+k^{\nu}\right)$ sufficiently small.

We observe first that if (a) holds, then (ii,a) gives that $\widehat{\mathscr{L}}_{M+1}$ has purely imaginary eigenvalues. Therefore, by (Riii), $U^{M+1}$ exists in $S_{h}$ as the unique solution of the linear system (4.5).

Suppose that (a) and (b) hold for some $M$ with $3 \leq M \leq J-1$. Then the assumptions of Proposition 4.1 are fulfilled and (4.23) takes the form

$$
\begin{aligned}
\left\|Q_{n+1} E^{n+1}\right\|_{n+1} \leq & \left(1+C_{*} k\right)\left\|Q_{n} E^{n}\right\|_{n}+C_{*} k\left\|Q_{n+1} E^{n+1}\right\|_{n+1} \\
& +C_{*} k\left(\left\|Q_{n-1} \widehat{E}^{n-1}\right\|_{n-1}+\cdots+\left\|Q_{n-3} \widehat{E}^{n-3}\right\|_{n-3}\right) \\
& +C_{*} k\left(k^{\nu}+h^{r}\right)
\end{aligned}
$$

where the constant $C_{*}$ is independent of $h, k$ but also independent of $M$. In the sequel, $C_{*}$ will denote a positive constant, not necessarily the same at any two places, independent of $h, k$, and $M$.

Letting $\Psi^{n}=\left\|Q_{n} E^{n}\right\|_{n}$, we have for sufficiently small $k$ that

$$
\Psi^{n+1}-\Psi^{n} \leq C_{*} k\left(\Psi^{n}+\cdots+\Psi^{n-3}\right)+C_{*} k\left(k^{\nu}+h^{r}\right), \quad n=3, \ldots, M .
$$

Using now $\Psi^{j} \leq C_{*}\left(k^{\nu}+h^{r}\right), j=0, \ldots, 3$, and summing from $n=3$ to $M$, we get

$$
\Psi^{M+1} \leq C_{*}\left(k^{\nu}+h^{r}\right)+C_{*} k \sum_{j=0}^{M} \Psi^{j} .
$$

The discrete Gronwall lemma therefore gives that

$$
\Psi^{M+1} \leq C_{*}\left(k^{\nu}+h^{r}\right) \exp \left(C_{*} k M\right) \leq C_{*}\left(k^{\nu}+h^{r}\right) .
$$

Now (4.24) and (ii,b) give (a) for sufficiently small $h, k$. Choosing $\widehat{C}$ small enough, we get, using again (4.24) and (ii,b), that $\left|E_{1}^{M+1}\right|_{1, \infty} \leq k$, i.e., that (b) holds for $M+1$. Consequently, (a) and (b) hold for every $M, 3 \leq M \leq J$. Hence, (4.24) holds for every $M, 0 \leq M \leq J-1$. Now using (III), (ii,a), (iv,a,b), and the Poincare inequality, we complete the proof. 
Remark. We may choose the starting values as $U_{1}^{0}=w^{0}, U_{2}^{0}=P u^{1}$ and for $j=1,2,3$ :

$$
U_{1}^{j}=w^{0}+P \sum_{l=1}^{\nu-1} \frac{(j k)^{l}}{l !} u^{(l)}(0), \quad U_{2}^{j}=P \sum_{l=0}^{\nu-1} \frac{(j k)^{l}}{l !} u^{(l+1)}(0) .
$$

The terms $u^{(m)}(0)$ are computed in terms of the initial data, using the equation (1.1). With this choice we have $\left\|Q_{j} E^{j}\right\|_{j} \leq c\left(k^{\nu}+h^{r}\right)$. We omit the proof.

\section{APPROXIMATION PROPERTIES OF THE ELLIPTIC PROJECTION}

In this section we shall justify, under certain assumptions on $S_{h}$, the properties (iv,a,b), ( $\mathrm{v}$ ) of the nonlinear elliptic projection $W(t)$, which has been defined in $\S 1$ as the function in $S_{h}$ satisfying

$$
a(W(t), \chi)=a(u(t), \chi), \quad \forall \chi \in S_{h}, 0 \leq t \leq T .
$$

For the existence and uniqueness of $W$ and the validity of (iv,a), we refer to Dobrowolski and Rannacher [12] and Rannacher [19]. Also, it can be proved, Rannacher [20], following the analysis of Rannacher and Scott [21], that

$$
\|u-W\|_{1, \infty} \leq c h^{r-1} \text {. }
$$

In the sequel we shall use (RS) to avoid the restriction hypothesis $r-N / 2-1 \geq 0$ in the proof of (iv,b) for $j=1$. However, our proof of (iv,b) for $j \geq 2$ needs this assumption.

We proceed now to show (iv,b), (v) for $j=1$. Differentiating (5.1) with respect to $t$, we get

$$
\tilde{a}(W(t) ; \dot{W}(t), \chi)=\tilde{a}(u(t) ; \dot{u}(t), \chi), \quad \forall \chi \in S_{h}, 0 \leq t \leq T .
$$

For the $H^{1}$ norm estimate we first assume that $h$ is small enough to ensure that $\nabla W \in \mathbf{Z}$. Using (5.2), (ii,a), (S2b), and (iii,a), we obtain

$$
\begin{aligned}
M_{0}\|\nabla(\dot{W}-\dot{u})\|^{2} \leq & \tilde{a}(W ; \dot{W}-\dot{u}, \dot{W}-\dot{u}) \\
= & \tilde{a}(W ; \dot{W}-\dot{u}, P \dot{u}-\dot{u}) \\
& +[\tilde{a}(u ; \dot{u}, \dot{W}-P \dot{u})-\tilde{a}(W ; \dot{u}, \dot{W}-P \dot{u})] \\
\leq & c h^{r-1}\|\dot{W}-\dot{u}\|_{1}+c h^{r-1}\|P(\dot{W}-\dot{u})\|_{1} .
\end{aligned}
$$

Hence, since $\|P v\|_{1} \leq c\|v\|_{1}$ and $S_{h} \subset H_{0}^{1}$, we have

$$
\|\dot{W}-\dot{u}\|_{1} \leq c h^{r-1}, \quad r \geq 2 .
$$

The relations (iii,a) and (ii,a) give (v) for $j=0$. For $j=1$ we have, cf. [6],

$$
\begin{aligned}
\|\dot{W}\|_{1, \infty} & \leq\|\dot{W}-\dot{u}\|_{1, \infty}+\|\dot{u}\|_{1, \infty} \leq\|\dot{W}-\chi\|_{1, \infty}+\|\dot{u}-\chi\|_{1, \infty}+c \\
& \leq c h^{-N / 2}\left(\|\dot{W}-\dot{u}\|_{1}+\|\dot{u}-\chi\|_{1}\right)+\|\dot{u}-\chi\|_{1, \infty}+c \text { for any } \chi \in S_{h} .
\end{aligned}
$$

If the element $\phi \in S_{h}$ satisfies $\|\phi-\dot{u}\|_{1, \infty} \leq c h^{r-1}$, putting in the above relation $\chi=\phi$, we have

$$
\|\dot{W}\|_{1, \infty} \leq c h^{r-N / 2-1}+c .
$$

Hence, (v) holds for $j=1$ if $r-N / 2-1 \geq 0$.

In the sequel we consider the following linear boundary value problem: For given $g \in L^{2}(\Omega)^{N}$ let $v \in H_{0}^{1}$ be the solution of

$$
\tilde{a}(u ; v, \phi)=(g, \phi), \quad \forall \phi \in H_{0}^{1},
$$


where $u$ is the solution of (1.1). The problem (5.4) has a unique solution $v \in H_{0}^{1}$, which satisfies the elliptic regularity conditions, cf. [14],

$$
\|v\|_{k+2} \leq c\|g\|_{k}, \quad k \geq 0 .
$$

Now let $v_{h}$ be the solution of the following discrete problem in $S_{h}$ : Find $v_{h} \in S_{h}$ such that

$$
\tilde{a}\left(u ; v_{h}, \chi\right)=(g, \chi), \quad \forall \chi \in S_{h} ;
$$

then it is known that we have, cf., e.g., [12],

$$
\begin{gathered}
\left\|v-v_{h}\right\|+h\left\|v-v_{h}\right\|_{1} \leq C h^{r}\|v\|_{r}, \\
\left\|v-v_{h}\right\|_{\infty}+h\left\|v-v_{h}\right\|_{1, \infty} \leq C h^{r}|\log h|^{\theta(r)}\|v\|_{r, \infty},
\end{gathered}
$$

where $\theta(r)=0$ if $r>2$ and $\theta(r)=\frac{N}{4}+1>0$ if $r=2$.

We return now to the error $\dot{u}-\dot{W}$. For the proof of the $L^{2}$ estimate, let $\Psi$ be the solution of the boundary value problem

$$
\tilde{a}(u ; \Psi, \phi)=(\dot{u}-\dot{W}, \phi), \quad \forall \phi \in H_{0}^{1} .
$$

Then we have

$$
\|\dot{u}-\dot{W}\|^{2}=\tilde{a}(u ; \Psi, \dot{u}-\dot{W}) .
$$

Also, (5.2) gives $\tilde{a}(u ; \dot{u}-\dot{W}, \chi)=\tilde{a}(W ; \dot{W}, \chi)-\tilde{a}(u ; \dot{W}, \chi), \chi \in S_{h}$. Hence, if $\Psi_{h}$ is the solution of the discrete problem in $S_{h}$ corresponding to (5.6), we obtain

$$
\|\dot{u}-\dot{W}\|^{2}=\tilde{a}\left(u ; \Psi-\Psi_{h}, \dot{u}-\dot{W}\right)+\left[\tilde{a}\left(W ; \dot{W}, \Psi_{h}\right)-\tilde{a}\left(u ; \dot{W}, \Psi_{h}\right)\right] .
$$

From (5.3), (5.5a), and (5.4a) we see that

$$
\left|\tilde{a}\left(u ; \Psi-\Psi_{h}, \dot{u}-\dot{W}\right)\right| \leq c h\|\Psi\|_{2} h^{r-1} \leq c h^{r}\|\dot{u}-\dot{W}\| .
$$

For the estimation of the last term of (5.7) we first observe that

$$
\begin{aligned}
\mid \tilde{a}(u ; & \left.\dot{W}, \Psi_{h}\right)-\tilde{a}\left(W ; \dot{W}, \Psi_{h}\right) \mid \\
\leq & \left|\tilde{a}\left(u ; \dot{W}-\dot{u}, \Psi_{h}\right)-\tilde{a}\left(W ; \dot{W}-\dot{u}, \Psi_{h}\right)\right|+\left|\tilde{a}\left(u ; \dot{u}, \Psi_{h}\right)-\tilde{a}\left(W ; \dot{u}, \Psi_{h}\right)\right| \\
\leq & \left|\tilde{a}\left(u ; \dot{W}-\dot{u}, \Psi_{h}-\Psi\right)-\tilde{a}\left(W ; \dot{W}-\dot{u}, \Psi_{h}-\Psi\right)\right| \\
& +|\tilde{a}(u ; \dot{W}-\dot{u}, \Psi)-\tilde{a}(W ; \dot{W}-\dot{u}, \Psi)| \\
& +\left|\tilde{a}\left(u ; \dot{u}, \Psi_{h}-\Psi\right)-\tilde{a}\left(W ; \dot{u}, \Psi_{h}-\Psi\right)\right|+|\tilde{a}(u ; \dot{u}, \Psi)-\tilde{a}(W ; \dot{u}, \Psi)| \\
= & : a_{1}+a_{2}+a_{3}+a_{4} .
\end{aligned}
$$

Since $|u-W|_{1, \infty} \leq c$, we have as in the proof of (5.8),

$$
a_{1}=\left|\tilde{a}\left(u ; \dot{W}-\dot{u}, \Psi_{h}-\Psi\right)-\tilde{a}\left(W ; \dot{W}-\dot{u}, \Psi_{h}-\Psi\right)\right| \leq c h^{r}\|\dot{u}-\dot{W}\| .
$$

For the term $a_{2}$ we distinguish the following cases:

(a) $N=1$; then $\|\Psi\|_{1, \infty} \leq C\|\Psi\|_{2}$, and hence

$$
a_{2}=|\tilde{a}(u ; \dot{W}-\dot{u}, \Psi)-\tilde{a}(W ; \dot{W}-\dot{u}, \Psi)| \leq c h^{r}\|\dot{u}-\dot{W}\| .
$$

(b) $N=2,3$; then, using (RS), we obtain

$$
a_{2}=|\tilde{a}(u ; \dot{W}-\dot{u}, \Psi)-\tilde{a}(W ; \dot{W}-\dot{u}, \Psi)| \leq c h^{2 r-2}\|\Psi\|_{1} \leq c h^{r}\|\dot{u}-\dot{W}\| .
$$

Since $|\dot{u}|_{1, \infty} \leq c$, there holds

$$
a_{3}=\left|\tilde{a}\left(u ; \dot{u}, \Psi_{h}-\Psi\right)-\tilde{a}\left(W ; \dot{u}, \Psi_{h}-\Psi\right)\right| \leq c h^{r}\|\Psi\|_{2} \leq c h^{r}\|\dot{u}-\dot{W}\| .
$$


For the estimation of $a_{4}$ we shall use the following Taylor formula: For $v, w \in \mathbf{Z}$ there holds

$$
\begin{aligned}
\tilde{a}(v ; \phi, \psi)-\tilde{a}(w ; \phi, \psi) & \\
= & \sum_{i, \alpha, j, \beta, k, \gamma=1}^{N}\left(\partial_{\gamma}\left(v_{k}-w_{k}\right) \partial_{\beta} \phi_{j} \frac{\partial A_{i \alpha j \beta}}{\partial \eta_{k \gamma}}(\nabla w), \partial_{\alpha} \psi_{i}\right) \\
& +\sum_{i, \alpha, j, \beta, k, \gamma, l, \delta=1}^{N}\left(\partial_{\delta}\left(v_{l}-w_{l}\right) \partial_{\gamma}\left(v_{k}-w_{k}\right) \partial_{\beta} \phi_{j}\right. \\
& \left.\times \int_{0}^{1} \frac{\partial^{2} A_{i \alpha j \beta}}{\partial \eta_{l \delta} \partial \eta_{k \gamma}}(\nabla w+\tau \nabla(v-w)) d \tau, \partial_{\alpha} \psi_{i}\right) \\
= & : b(v, w, \phi, \psi)+d(v, w, \phi, \psi) .
\end{aligned}
$$

Taking $h$ sufficiently small to ensure that $\nabla W \in \mathbf{Z}$, we apply the above formula for $W, \dot{u}, \Psi$ and $u, \dot{u}, \Psi$ :

$$
a_{4}=|\tilde{a}(W ; \dot{u}, \Psi)-\tilde{a}(u ; \dot{u}, \Psi)|=|b(W, u, \dot{u}, \Psi)+d(W, u, \dot{u}, \Psi)| .
$$

For the estimation of $b(W, u, \dot{u}, \Psi)$ we use the Gauss-Green Theorem and the fact that $S_{h} \subset H_{0}^{1}$, and obtain

$$
\begin{aligned}
|b(W, u, \dot{u}, \Psi)| & =\left|\int_{\Omega_{i, \alpha, j, \beta, k, \gamma=1}} \sum_{i}^{N}\left\{\partial_{\gamma}\left[\partial_{\beta} \dot{u}_{j} \frac{\partial A_{i \alpha j \beta}}{\partial \eta_{k \gamma}}(\nabla u), \partial_{\alpha} \Psi_{i}\right]\left(W_{k}-u_{k}\right)\right\} d x\right| \\
& \leq C\|\dot{u}\|_{2, \infty}\|u\|_{2, \infty}\|\Psi\|_{2}\|W-u\| .
\end{aligned}
$$

Since $u$ is smooth enough, using (5.4a) and (iii,a), we obtain

$$
|b(W, u, \dot{u}, \Psi)| \leq c h^{r}\|\dot{u}-\dot{W}\| .
$$

In the same way as before we estimate the term $d(W, u, \dot{u}, \Psi)$ : In particular, if

(a) $N=1$, then, since $\|\Psi\|_{1, \infty} \leq C\|\Psi\|_{2}$, we have

$$
|d(W, u, \dot{u}, \Psi)| \leq c h^{r}\|\dot{u}-\dot{W}\|,
$$

and if

(b) $N=2,3$, using (RS), we see that

$$
|d(W, u, \dot{u}, \Psi)| \leq c h^{2 r-2}\|\Psi\|_{1} \leq c h^{r}\|\dot{u}-\dot{W}\| .
$$

Summarizing the above estimations for $a_{1}, a_{2}, a_{3}$, and $a_{4}$, we obtain

$$
\left|\tilde{a}\left(W ; \dot{W}, \Psi_{h}\right)-\tilde{a}\left(u ; \dot{W}, \Psi_{h}\right)\right| \leq c h^{r}\|\dot{u}-\dot{W}\| .
$$

Combining (5.7), (5.8), and (5.9), we finally conclude

$$
\|\dot{u}-\dot{W}\| \leq c h^{r},
$$

which is (iv,b) for $j=1$. The proof for $j \geq 2$ is given in [18], [16].

\section{ACKNOWLEDGMENTS}

The results of this work are taken from author's doctoral thesis [16]. The author would like to thank his advisor Professor Vassilios A. Dougalis for his guidance and many helpful suggestions regarding this work. The author also thanks Professor Georgios D. Akrivis for contributing many valuable remarks. 


\section{BIBLIOGRAPHY}

1. G. A. Baker and J. H. Bramble, Semidiscrete and fully discrete approximations for second-order hyperbolic equations, RAIRO Anal. Numér. 13 (1979), 75-100.

2. G. A. Baker, V. A. Dougalis, and S. M. Serbin, High-order two-step approximations for hyperbolic equations, RAIRO Anal. Numér. 13 (1979), 201-226.

3. L. A. Bales, Semidiscrete and single step fully discrete approximations for second order hyperbolic equations with time-dependent coefficients, Math. Comp. 43 (1984), 383-414.

4. _ High-order single-step fully discrete approximations for nonlinear second order hyperbolic equations, Comput. Math. Appl. 12A (1986), 581-604.

5. L. A. Bales, V. A. Dougalis, and S. M. Serbin, Cosine methods for second-order hyperbolic equations with time-dependent coefficients, Math. Comp. 45 (1985), 65-89.

6. L. A. Bales and V. A. Dougalis, Cosine methods for nonlinear second-order hyperbolic equations, Math. Comp. 52 (1989), 299-319, S15-S33.

7. J. H. Bramble and P. H. Sammon, Efficient high-order single-step methods for parabolic problems: Part II, unpublished manuscript.

8. V. C. Chen and W. von Wahl, Das Rand-Anfangswertproblem für quasilineare Wellengleichungen in Sobolevräumen niedriger Ordnung, J. Reine Angew. Math. 337 (1982), 77112.

9. P. G. Ciarlet, The finite element method for elliptic problems, North-Holland, Amsterdam, 1978.

10. C. M. Dafermos and W. J. Hrusa, Energy methods for quasilinear hyperbolic initial-boundary value problems. Applications to elastodynamics, Arch. Rational Mech. Anal. 87 (1985), 267-292.

11. J. E. Dendy, Jr., Galerkin's method for some highly nonlinear problems, SIAM J. Numer. Anal. 14 (1977), 327-347.

12. M. Dobrowolski and R. Rannacher, Finite element methods for nonlinear elliptic systems of second order, Math. Nachr. 94 (1980), 155-172.

13. T. Dupont, $L^{2}$-estimates for Galerkin methods for second-order hyperbolic equations, SIAM J. Numer. Anal. 10 (1973), 880-889.

14. G. Fichera, Existence theorems in elasticity, Encyclopedia of Physics, vol. VI a/2 (C. Truesdell, ed.), Springer-Verlag, Berlin, Heidelberg, New York, 1972, pp. 347-389.

15. M. E. Gurtin, An introduction to continuum mechanics, Academic Press, New York, 1981.

16. Ch. G. Makridakis, Galerkin/finite element methods for the equations of elastodynamics, Ph.D. thesis, Univ. of Crete, 1989. (Greek)

17. _ Cosine methods for a class of semilinear second-order wave equations, Comput. Math. Appl. 19 (1990), 19-34.

18. __ Finite element approximations of nonlinear elastic waves, Technical Report, Dept. of Mathematics, Univ. of Crete, 1992.

19. R. Rannacher, On finite element approximation of general boundary value problems in nonlinear elasticity, Calcolo 17 (1980), 175-193.

20. $ـ$, Private communication, 1988.

21. R. Rannacher and R. Scott, Some optimal error estimates for piecewise linear finite element approximations, Math. Comp. 38 (1982), 437-470.

Department of Mathematics, University of Crete, Iraklion, Greece

Current address: Department of Mathematics, University of Maryland, College Park, Maryland 20742

E-mail address: makr@talos.cc.uch.gr 